\title{
THE HE-RICH CORE-COLLAPSE SUPERNOVA 2007Y: OBSERVATIONS FROM X-RAY TO RADIO WAVELENGTHS ${ }^{*, \dagger}$
}

\author{
Maximilian Stritzinger ${ }^{1,2}$, Paolo Mazzali $^{3,4}$, Mark M. Phillips ${ }^{1}$, Stefan Immler $^{5,6}$, Alicia Soderberg $^{7,8}$, \\ Jesper Sollerman $^{2,9}$, Luis Boldt ${ }^{1}$, Jonathan Braithwaite ${ }^{10}$, Peter Brown ${ }^{11}$, Christopher R. Burns ${ }^{12}$, \\ Carlos Contreras $^{1}{ }^{\text {, Ricardo Covarrubias }}{ }^{1}$, Gastón Folatelli ${ }^{13}$, Wendy L. Freedman ${ }^{12}$, Sergio González ${ }^{1}$, \\ Mario Hamuy ${ }^{13}$, Wojtek Krzeminski ${ }^{1}$, Barry F. Madore ${ }^{12,14}{ }^{\text {, Peter Milne }}{ }^{15}$, Nidia Morrell ${ }^{1}$, S. E. Persson ${ }^{12}$, \\ Miguel Roth ${ }^{1}$, Mathew Smith ${ }^{16}$, and Nicholas B. SuntzefF ${ }^{17}$ \\ ${ }^{1}$ Las Campanas Observatory, Carnegie Observatories, Casilla 601, La Serena, Chile; mstritzinger@1co.cl, mmp@1co.cl, 1boldt@1co.cl, ccontreras@1co.cl, \\ ricardo@1co.cl,sgonzalez@1co.cl,wojtek@1co.cl,nmorrell@1co.cl, miguel@1co.cl \\ ${ }^{2}$ Dark Cosmology Centre, Niels Bohr Institute, University of Copenhagen, Juliane Maries Vej 30, 2100 Copenhagen $\emptyset$, Denmark; max@ dark-cosmology.dk, \\ jesper@dark-cosmology.dk \\ ${ }^{3}$ Max-Planck-Institut für Astrophysik, Karl-Schwarzschild-Str. 1, 85741 Garching bei München, Germany; mazzali@mpa-garching.mpg.de \\ ${ }^{4}$ INAF-Osservatorio Astronomico di Padova, vicolo dell'Osservatorio 5, 35122 Padova, Italy \\ ${ }^{5}$ Astrophysics Science Division, X-RayAstrophysical Laboratory, Code662, NASA Goddard Space Flight Center, Greenbelt, MD 20771, USA; \\ stefan.m.immler@nasa.gov \\ ${ }^{6}$ Universities Space Research Association, 10211 Wincopin Circle, Columbia, MD 21044, USA \\ ${ }^{7}$ Department of Astrophysical Sciences, Princeton University, Ivy Lane, Princeton, New Jersey 08544, USA; alicia@ astro.princeton.edu \\ ${ }^{8}$ Harvard-Smithsonian Center for Astrophysics, 60 Garden Street, Cambridge, MA 02138, USA \\ ${ }^{9}$ Department of Astronomy, Stockholm University, AlbaNova, SE-10691 Stockholm, Sweden \\ ${ }^{10}$ Canadian Institute for Theoretical Astrophysics, 60 St. George St., Toronto M5S 3H8, Canada; jon@ @ita.utoronto.ca \\ ${ }^{11}$ Pennsylvania State University, Department of Astronomy \& Astrophysics, University Park, PA 16802, USA; pbrown@ astro.psu.edu \\ 12 Observatories of the Carnegie Institution of Washington, 813 Santa Barbara St., Pasadena, CA 91101, USA; cburns@ @ociw.edu, wendy@ ociw.edu, \\ barry@ociw.edu, persson@ociw.edu \\ ${ }^{13}$ Universidad de Chile, Departamento de Astronomía, Casilla 36-D, Santiago, Chile; gaston@ das.uchile.cl, mhamuy@das.uchile.cl \\ ${ }^{14}$ Infrared Processing and Analysis Center, Caltech/Jet Propulsion Laboratory, Pasadena, CA 91125, USA \\ ${ }^{15}$ Department of Astronomy and Steward Observatory, University of Arizona, Tucson, AZ 85721, USA; pmilne@ as.asrizona.edu \\ ${ }^{16}$ Cosmology and Gravity Group, Department of Mathematics and Applied Mathematics, University of Cape Town, South Africa; mathew.smith@uct.ac.za \\ 17 Texas A\&M University, Physics Department, College Station, TX 77843, USA; nsuntzeff@tamu.edu \\ Received 2009 January 18; accepted 2009 February 12; published 2009 April 17
}

\begin{abstract}
A detailed study spanning approximately a year has been conducted on the Type Ib supernova (SN) 2007Y. Imaging was obtained from X-ray to radio wavelengths, and a comprehensive set of multi-band $\left(w 2 m 2 w 1 u^{\prime} g^{\prime} r^{\prime} i^{\prime} U B V Y J H K_{s}\right)$ light curves and optical spectroscopy is presented. A virtually complete bolometric light curve is derived, from which we infer a ${ }^{56} \mathrm{Ni}$ mass of $0.06 M_{\odot}$. The early spectrum strongly resembles SN 2005bf and exhibits high-velocity features of $\mathrm{Ca}$ II and $\mathrm{H} \alpha$; during late epochs the spectrum shows evidence of an ejecta-wind interaction. Nebular emission lines have similar widths and exhibit profiles that indicate a lack of major asymmetry in the ejecta. Late phase spectra are modeled with a non-LTE code, from which we find ${ }^{56} \mathrm{Ni}, \mathrm{O}$, and total-ejecta masses (excluding $\mathrm{He}$ ) to be $0.06,0.2$, and $0.42 M_{\odot}$, respectively, below $4500 \mathrm{~km} \mathrm{~s}^{-1}$. The ${ }^{56} \mathrm{Ni}$ mass confirms results obtained from the bolometric light curve. The oxygen abundance suggests that the progenitor was most likely a $\approx 3.3 M_{\odot}$ He core star that evolved from a zero-age-main-sequence mass of 10-13 $M_{\odot}$. The explosion energy is determined to be $\approx 10^{50} \mathrm{erg}$, and the mass-loss rate of the progenitor is constrained from X-ray and radio observations to be $\lesssim 10^{-6} M_{\odot} \mathrm{yr}^{-1}$. SN $2007 \mathrm{Y}$ is among the least energetic normal Type $\mathrm{Ib}$ SNe ever studied.
\end{abstract}

Key words: galaxies: individual (NGC 1187) - supernovae: general - supernovae: individual (SN 2007Y)

Online-only material: color figures

\section{INTRODUCTION}

A decade has passed since it was first recognized that some extragalactic gamma-ray transients could be linked to the death of massive stars (Galama et al. 1998). Soon afterward, the connection between long gamma-ray bursts (GRBs) and energetic Type Ic supernovae (SNe Ic) was firmly established (Hjorth et al. 2003; Matheson et al. 2003; Stanek et al. 2003; Malesani et al. 2004). Over the last several years it has also become clear that some $\mathrm{X}$-ray flashes, a less energetic version

\footnotetext{
* This paper includes data gathered with the $6.5 \mathrm{~m}$ Magellan telescope at Las Campanas Observatory, Chile.

$\dagger$ Partly based on observations collected at the European Southern Observatory, La Silla and Paranal Observatories, Chile (ESO Programme 078.D-0048 and 380.D-0272).
}

of GRBs, are produced by a similar type of SN Ic (Fynbo et al. 2004; Modjaz et al. 2006; Pian et al. 2006; Sollerman et al. 2006).

Recently, X-ray emission was detected from the He-rich Type Ib SN 2008D (Soderberg et al. 2008; Mazzali et al. 2008; Malesani et al. 2009; Modjaz et al. 2009). This finding has given fresh impetus to the study of normal $\mathrm{SNe} \mathrm{Ib}$, which has been somewhat neglected, only a handful of events having been well observed. In this paper, we report detailed observations, obtained through the course of the Carnegie Supernova Project (here after CSP; Hamuy et al. 2006), of the Type Ib SN 2007Y. The earliest spectra of this event bear a striking resemblance to the unusual Type Ib SN 2005bf (Anupama et al. 2005; Folatelli et al. 2006); however, its light curves exhibit a more ordinary evolution. 


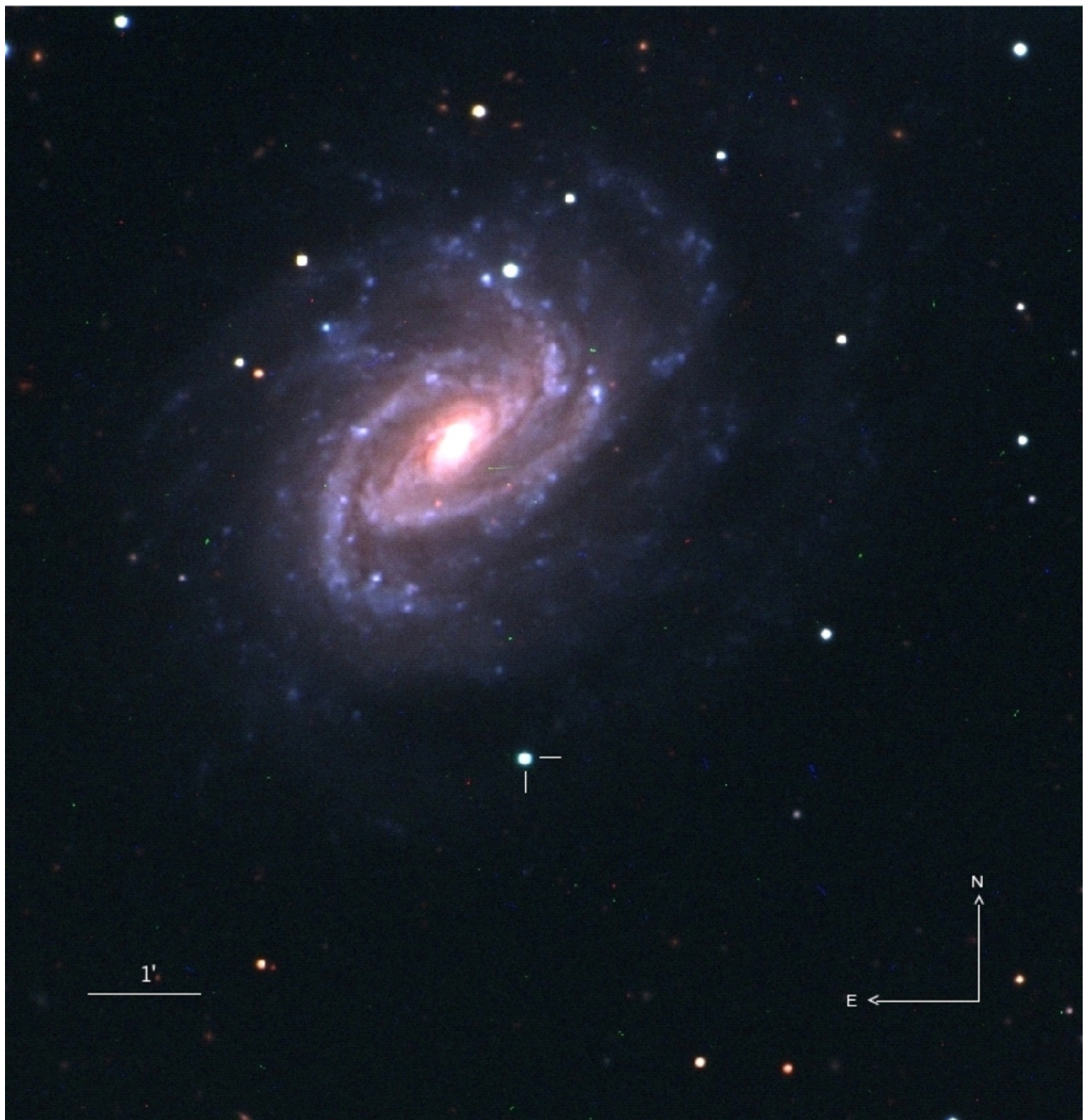

Figure 1. Color image of NGC 1187 with SN 2007Y indicated.

(A color version of this figure is available in the online journal.)

SN 2007Y (see Figure 1) was discovered in the nearby spiral galaxy NGC 1187 on February 15.77 UT $^{18}$ (Monard 2007), and was initially classified as a young peculiar $\mathrm{SN} \mathrm{Ib/c} \mathrm{(Folatelli}$ et al. 2007). As this was a young and nearby SN it made an excellent target for our follow-up program.

In this paper, a comprehensive set of X-ray, ultraviolet, optical, near-infrared, and radio observations of SN 2007Y are presented to gain insight into the explosion physics, and determine the stellar evolutionary path of the progenitor. X-ray and ultraviolet observations were carried out with the X-Ray Telescope (XRT) and the UltraViolet Optical Telescope (UVOT) aboard the Swift satellite (Roming et al. 2005). Early-phase spectroscopy and imaging was collected at Las Campanas Observatory (LCO). Late-time spectroscopy and imaging came from facilities at LCO and at the European Organisation for Astronomical Research in the Southern Hemisphere's (ESO) Paranal and La Silla Observatories. Finally, our radio observations were obtained with the Very Large Array. ${ }^{19}$

In Section 2, a concise description of the observations is given. More complete details regarding the data reduction methods applied to the optical and near-infrared observations can be

\footnotetext{
${ }^{18}$ Universal Time (UT) is used throughout this paper.

19 The Very Large Array is a facility of the National Science Foundation operated under cooperative agreement by Associated Universities, Inc.
}

found in the Appendix. Section 3 contains the analysis of the data, while the discussion is presented in Section 4. We conclude with a summary in Section 5.

\section{OBSERVATIONS}

Six weeks of imaging was obtained at LCO covering the flux evolution from -14 to +41 days past maximum light. ${ }^{20}$ Optical imaging was performed with a set of Sloan $u^{\prime} g^{\prime} r^{\prime} i^{\prime}$ and Johnson $B$ and $V$ filters, while in the near-infrared a set of $J H K_{s}$ filters identical to those used by Persson et al. (1998) were employed. The $Y$ band is defined in Hillenbrand et al. (2002), and details concerning its calibration are given in Hamuy et al. (2006, hereafter Paper I) and C. Contreras et al. (2009, in preparation). In addition, two epochs of optical and near-infrared imaging were conducted at late epochs with the VLT.

Nineteen epochs of early phase photometry obtained with the UVOT and originally published in Brown et al. (2008) are also included in our analysis. These data were obtained with $w 2 m 2 w 1 U B V$ passbands and are critical to calculating a nearly complete bolometric light curve. SN 2007Y was also observed with XRT. No X-ray emission was detected, but these

\footnotetext{
${ }^{20}$ Maximum light refers to the time of peak bolometric brightness $\left(L_{\max }\right)$, i.e., March 3.5 or JD-2454163.12 (see Section 3.4).
} 


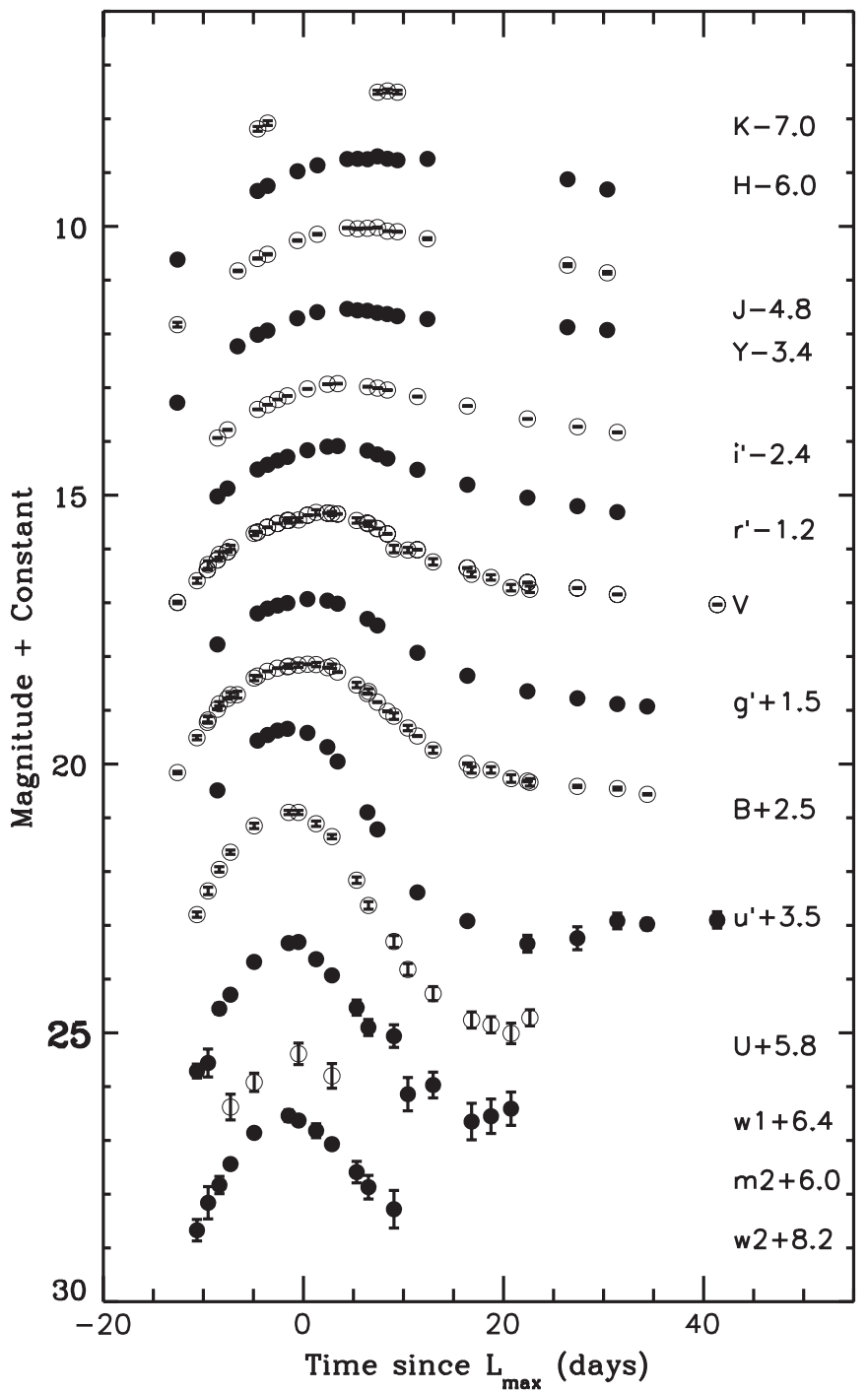

Figure 2. Observed ultraviolet, optical, and near-infrared early-time light curves of SN 2007Y plotted as a function of time since bolometric maximum. The light curves have been shifted in the $y$-direction for clarity.

observations allow us to place an upper limit on the mass-loss rate of the progenitor prior to explosion.

Early and late phase radio monitoring spanning from -8 to 653 days past maximum were obtained with the VLA. With these observations constraints are placed on the mass-loss rate of the progenitor star and the interaction of circumstellar material (CSM) with the SN shock wave. Our radio observations are complementary to the XRT observations and provide a more stringent limit on the mass-loss rate.

Table 1 contains the positions and average magnitudes of the optical and near-infrared local sequence stars used to compute final photometry from the CSP images. The definitive optical and near-infrared photometry in the standard Landolt (1992) $(B V)$ and Smith et al. (2002) $\left(u^{\prime} g^{\prime} r^{\prime} i^{\prime}\right)$ systems are given in Tables 2 and 3, respectively.

Early phase ultraviolet, optical, and near-infrared light curves of SN 2007Y are shown in Figure 2. Late phase photometry is combined with the corresponding early epoch light curves in Figure 3. Note the VLT optical images were taken with a set of facility Johnson Kron-Cousins BVRI and the near-infrared images with $J_{s} H K_{s}$ filters (Persson et al. 1998).

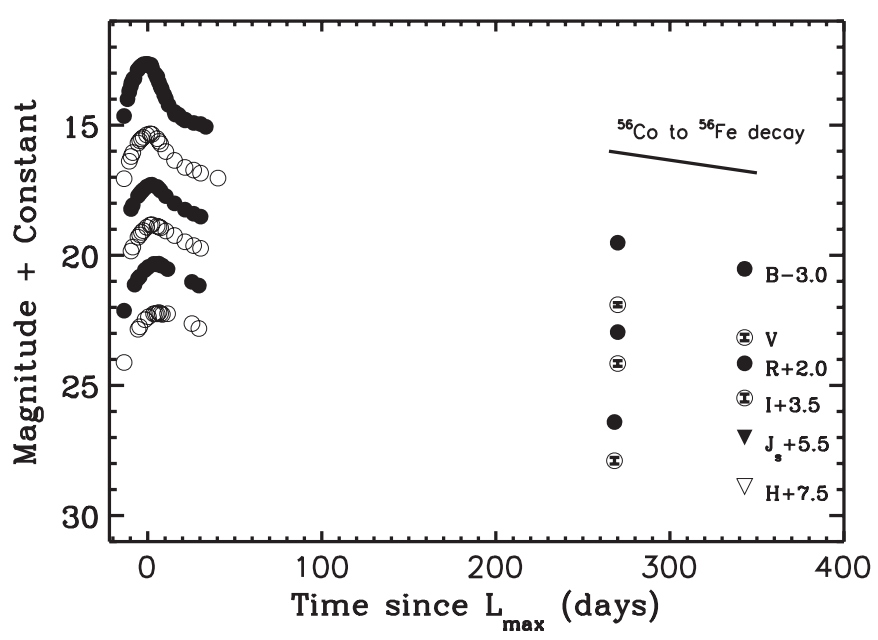

Figure 3. Late phase VLT $B V R I J_{s} H$ photometry plotted with early-time $B V r^{\prime} i^{\prime} J H$ light curves vs. time since $L_{\max }$. The light curves have been shifted in the $y$-direction for clarity as indicated. Up side down triangles are $3 \sigma$ upper limits.

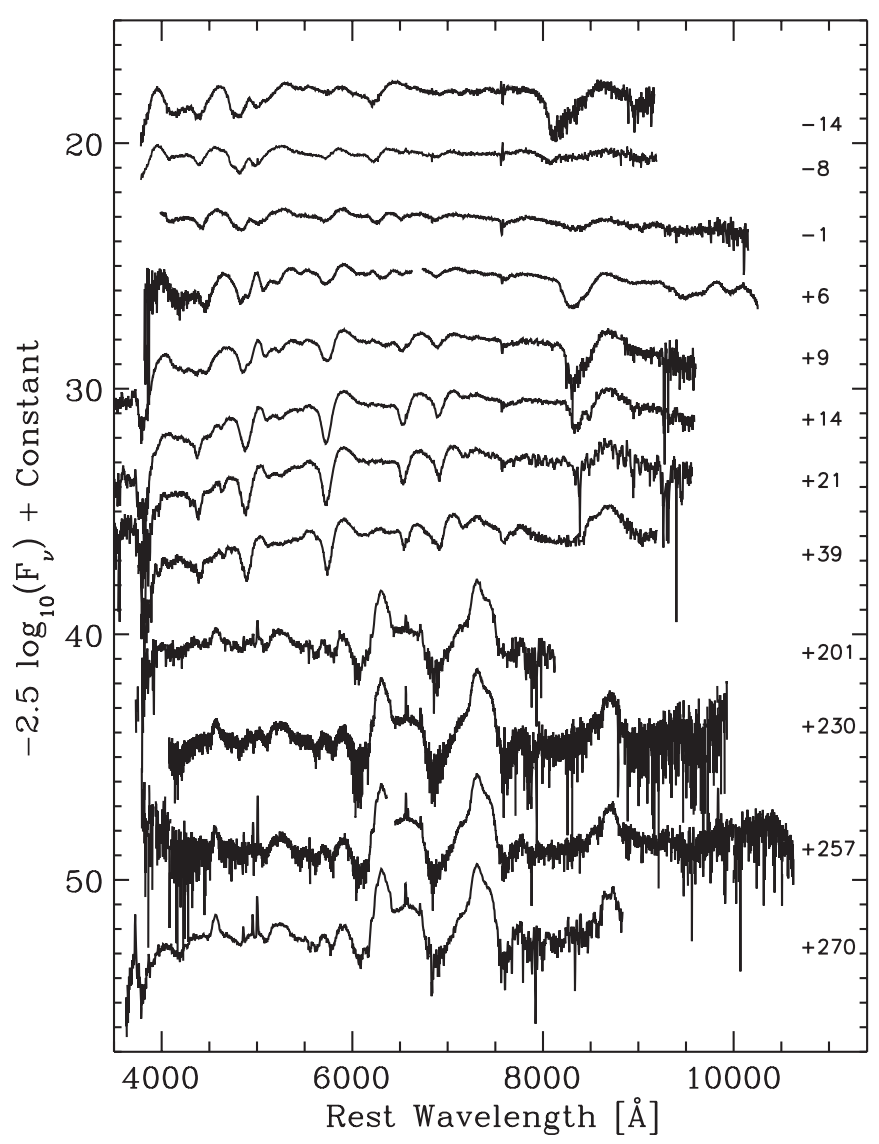

Figure 4. Spectroscopic sequence of SN 2007Y ranging from -14 to +270 days past $L_{\max }$. Each spectrum is plotted in a logarithmic $\left(f_{v}\right)$ scale. The spectra have been shifted below the day -14 spectrum by an arbitrary amount for presentation. The labels on the right indicate the phase with respect to $L_{\max }$. Each spectrum has been corrected to the rest frame of SN 2007Y adopting the redshift $z=0.00463$.

A total of 12 epochs of long slit optical spectroscopy were collected over the course of $\sim 300$ days. Table 4 contains the journal of spectroscopic observations and gives details concerning the telescope, instrument setup, and final data product. The spectra corrected to the rest frame of the host galaxy are presented in Figure 4. For clarity each spectrum has 
Table 1

Photometry of the Local Sequence Stars in the Field of NGC 1187

\begin{tabular}{|c|c|c|c|c|c|c|c|c|c|c|c|c|}
\hline $\begin{array}{l}\text { Star } \\
\text { ID }\end{array}$ & R.A. & Decl. & $u^{\prime}$ & $g^{\prime}$ & $r^{\prime}$ & $i^{\prime}$ & $B$ & V & $Y$ & $J$ & $H$ & $K_{s}$ \\
\hline$\overline{\mathrm{C} 1}$ & 45.72981 & -22.85382 & $19.068(054)$ & $16.163(015)$ & $\ldots$ & $\ldots$ & 16.881(013) & $\ldots$ & $\ldots$ & $\ldots$ & $\ldots$ & $\ldots$ \\
\hline $\mathrm{C} 2$ & 45.59832 & -22.83040 & $17.267(011)$ & $15.961(007)$ & $15.449(009)$ & $15.244(009)$ & 16.362(009) & $15.668(008)$ & $\ldots$ & $\ldots$ & $\ldots$ & $\ldots$ \\
\hline $\mathrm{C} 3$ & 45.69047 & -22.82723 & $17.329(012)$ & $16.114(007)$ & $15.683(008)$ & $15.520(008)$ & $16.489(011)$ & $15.861(007)$ & $\ldots$ & $\ldots$ & $\ldots$ & $\ldots$ \\
\hline $\mathrm{C} 4$ & 45.65395 & -22.83600 & $19.246(077)$ & $16.630(013)$ & $15.415(008)$ & $14.873(007)$ & 17.292(015) & $15.996(011)$ & $\ldots$ & $\ldots$ & $\ldots$ & $\ldots$ \\
\hline C5 & 45.60573 & -22.93198 & $17.840(014)$ & $16.669(007)$ & $16.254(009)$ & $16.093(009)$ & $17.023(016)$ & $16.422(007)$ & $\ldots$ & $\ldots$ & $\ldots$ & $\ldots$ \\
\hline C6 & 45.67210 & -22.84998 & $19.925(092)$ & 17.192(007) & $15.967(010)$ & $15.440(007)$ & 17.878(014) & $16.547(012)$ & $\ldots$ & $\ldots$ & $\ldots$ & $\ldots$ \\
\hline $\mathrm{C} 7$ & 45.61845 & -22.88566 & $18.495(024)$ & $16.989(012)$ & $16.450(011)$ & $16.256(007)$ & $17.419(019)$ & $16.683(012)$ & $\ldots$ & $\ldots$ & $\ldots$ & $\ldots$ \\
\hline $\mathrm{C} 8$ & 45.59823 & -22.86743 & $18.666(033)$ & $17.391(007)$ & $16.907(007)$ & $16.725(009)$ & 17.783(017) & $17.099(010)$ & $\ldots$ & $\ldots$ & $\ldots$ & $\ldots$ \\
\hline C9 & 45.58725 & -22.93928 & $19.183(091)$ & $17.521(011)$ & $16.859(033)$ & $16.571(011)$ & $18.025(031)$ & $17.131(025)$ & $\ldots$ & $\ldots$ & $\ldots$ & $\ldots$ \\
\hline $\mathrm{C} 10$ & 45.64463 & -22.84426 & $19.173(047)$ & $17.583(008)$ & $16.966(009)$ & $16.720(012)$ & 18.053(019) & $17.233(018)$ & $\ldots$ & $\ldots$ & $\ldots$ & $\ldots$ \\
\hline $\mathrm{C} 11$ & 45.61671 & -22.85780 & $19.763(087)$ & $17.742(015)$ & $16.944(010)$ & 16.643(007) & 18.251(014) & $17.307(019)$ & $\ldots$ & $\ldots$ & $\ldots$ & $\ldots$ \\
\hline $\mathrm{C} 12$ & 45.62911 & -22.84027 & $18.581(028)$ & $17.687(008)$ & $17.385(008)$ & $17.283(011)$ & $17.975(016)$ & $17.514(009)$ & $\ldots$ & $\ldots$ & $\ldots$ & $\ldots$ \\
\hline $\mathrm{C} 13$ & 45.67855 & -22.85963 & $19.859(086)$ & $17.862(009)$ & $17.145(009)$ & $16.857(010)$ & 18.353(018) & $17.483(008)$ & $\ldots$ & $\ldots$ & $\ldots$ & $\ldots$ \\
\hline $\mathrm{C} 14$ & 45.62110 & -22.94564 & $20.726(185)$ & $18.239(014)$ & 16.897(011) & $16.162(008)$ & $19.012(026)$ & $17.530(010)$ & $15.018(027)$ & $14.612(017)$ & $13.996(034)$ & \\
\hline $\mathrm{C} 15$ & 45.63166 & -22.92610 & $20.736(193)$ & $18.483(029)$ & $17.140(013)$ & $16.445(007)$ & $19.128(058)$ & $17.757(011)$ & $15.334(017)$ & $14.939(013)$ & $14.328(050)$ & $14.157(059)$ \\
\hline $\mathrm{C} 16$ & 45.59841 & -22.85483 & $20.471(150)$ & $18.473(017)$ & $17.810(008)$ & $17.563(009)$ & 18.983(025) & $18.125(011)$ & $\ldots$ & $\ldots$ & $\ldots$ & $\ldots$ \\
\hline $\mathrm{C} 17$ & 45.59876 & -22.91843 & $21.148(362)$ & $18.887(042)$ & $17.529(009)$ & $16.738(014)$ & $19.622(044)$ & 18.141(016) & $\ldots$ & $\ldots$ & $\ldots$ & $\ldots$ \\
\hline $\mathrm{C} 18$ & 45.58554 & -22.85900 & $21.906(830)$ & $18.935(034)$ & $17.621(018)$ & $16.868(011)$ & $19.543(115)$ & $18.235(046)$ & $\ldots$ & $\ldots$ & $\ldots$ & $\ldots$ \\
\hline C19 & 45.67666 & -22.91654 & $21.821(535)$ & $18.908(025)$ & $17.592(007)$ & $16.481(007)$ & $19.708(048)$ & $18.194(015)$ & $15.099(016)$ & $14.611(022)$ & $14.087(040)$ & $13.867(063)$ \\
\hline $\mathrm{C} 20$ & 45.62264 & -22.92669 & $22.066(983)$ & $19.108(030)$ & $17.774(009)$ & 16.481(007) & $20.005(068)$ & $18.353(013)$ & $14.993(050)$ & $14.527(013)$ & $14.001(052)$ & $13.685(048)$ \\
\hline $\mathrm{C} 21$ & 45.61848 & -22.88557 & $\ldots$ & $\ldots$ & $\ldots$ & $\ldots$ & $\ldots$ & $\ldots$ & $15.532(063)$ & $15.281(012)$ & $14.954(048)$ & $14.811(122)$ \\
\hline $\mathrm{C} 22$ & 45.73816 & -22.90095 & $\ldots$ & $\ldots$ & $\ldots$ & $\ldots$ & $\ldots$ & $\ldots$ & $16.023(026)$ & $15.605(015)$ & $15.038(067)$ & $\ldots$ \\
\hline $\mathrm{C} 23$ & 45.70857 & -22.96788 & $\ldots$ & $\ldots$ & $\ldots$ & $\ldots$ & $\ldots$ & $\ldots$ & $16.434(049)$ & $16.002(023)$ & $15.547(033)$ & $\ldots$ \\
\hline $\mathrm{C} 24$ & 45.71329 & -22.93300 & $\ldots$ & $\ldots$ & $\ldots$ & $\ldots$ & $\ldots$ & $\ldots$ & $16.930(041)$ & $16.321(035)$ & $15.848(062)$ & $\ldots$ \\
\hline $\mathrm{C} 25$ & 45.73165 & -22.92826 & $\ldots$ & $\ldots$ & $\ldots$ & $\ldots$ & $\ldots$ & $\ldots$ & $16.641(033)$ & $16.146(030)$ & $15.629(054)$ & $\ldots$ \\
\hline $\mathrm{C} 26$ & 45.71723 & -22.92116 & $\ldots$ & $\ldots$ & $\ldots$ & $\ldots$ & $\ldots$ & $\ldots$ & $16.803(076)$ & $16.322(038)$ & $15.844(064)$ & 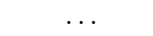 \\
\hline $\mathrm{C} 27$ & 45.70581 & -22.95958 & $\ldots$ & $\ldots$ & $\ldots$ & $\ldots$ & $\ldots$ & $\ldots$ & $16.961(051)$ & $16.522(036)$ & $16.065(062)$ & . \\
\hline $\mathrm{C} 28$ & 45.63538 & -22.93916 & $\ldots$ & $\ldots$ & $\ldots$ & $\ldots$ & $\ldots$ & $\ldots$ & $17.184(065)$ & $16.611(079)$ & $16.317(094)$ & $\ldots$ \\
\hline $\mathrm{C} 29$ & 45.67655 & -22.86069 & $\ldots$ & $\ldots$ & $\ldots$ & $\ldots$ & $\ldots$ & $\ldots$ & $15.303(023)$ & $14.915(022)$ & $14.278(027)$ & $\ldots$ \\
\hline
\end{tabular}

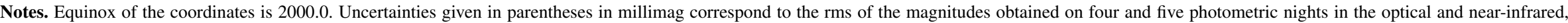
respectively. The magnitudes of the four $K_{s}$-band stars are the standard values from the 2MASS catalog. 
Table 2

CSP Optical Photometry of SN 2007Y

\begin{tabular}{|c|c|c|c|c|c|c|c|}
\hline JD-2,400,000 & $u^{\prime}$ & $g^{\prime}$ & $r^{\prime}$ & $i^{\prime}$ & $B$ & $V$ & Instrument \\
\hline 54150.53 & $\ldots$ & $\ldots$ & $\ldots$ & $\ldots$ & $17.658(024)$ & $16.994(024)$ & Tek 5 \\
\hline 54153.51 & $\ldots$ & $\ldots$ & $\ldots$ & $\ldots$ & $16.724(015)$ & $16.386(015)$ & Site 3 \\
\hline 54154.51 & $16.990(015)$ & $16.275(015)$ & $16.222(015)$ & $16.336(015)$ & $16.481(015)$ & $16.205(015)$ & Site 3 \\
\hline 54155.52 & $\cdots$ & $\cdots$ & $16.075(015)$ & $16.184(015)$ & $16.282(015)$ & $16.054(015)$ & Site 3 \\
\hline 54156.51 & $\ldots$ & $\ldots$ & $\ldots$ & $\ldots$ & $16.210(064)$ & $\ldots$ & Tek 5 \\
\hline 54158.52 & $16.064(015)$ & $15.700(015)$ & $15.722(015)$ & $15.804(015)$ & $15.864(015)$ & $15.687(015)$ & Site 3 \\
\hline 54159.52 & $15.960(015)$ & $15.608(015)$ & $15.634(015)$ & $15.717(015)$ & $15.775(015)$ & $15.596(015)$ & Site 3 \\
\hline 54160.51 & $15.877(016)$ & $15.552(015)$ & $15.553(015)$ & $15.620(015)$ & $15.717(015)$ & $15.526(015)$ & Site 3 \\
\hline 54161.50 & $15.845(021)$ & $15.506(015)$ & $15.487(015)$ & $15.552(015)$ & $15.692(015)$ & $15.469(015)$ & Site 3 \\
\hline 54163.51 & $15.921(015)$ & $15.432(015)$ & $15.364(015)$ & $15.423(015)$ & $15.644(015)$ & $15.372(015)$ & Site 3 \\
\hline 54165.50 & $16.183(016)$ & $15.461(015)$ & $15.297(015)$ & $15.336(015)$ & $15.707(015)$ & $15.332(015)$ & Site 3 \\
\hline 54166.52 & $16.452(015)$ & $15.518(015)$ & $15.286(015)$ & $15.325(015)$ & $15.789(015)$ & $15.349(015)$ & Site 3 \\
\hline 54169.50 & $17.398(015)$ & $15.800(015)$ & $15.370(015)$ & $15.382(015)$ & $16.188(015)$ & $15.519(015)$ & Site 3 \\
\hline 54170.50 & $17.716(016)$ & $15.923(015)$ & $15.442(015)$ & $15.408(015)$ & $16.349(015)$ & $15.620(015)$ & Site 3 \\
\hline 54171.51 & $\cdots$ & $\ldots$ & $15.517(015)$ & $15.444(015)$ & $16.520(015)$ & $15.719(015)$ & Site 3 \\
\hline 54174.50 & $18.887(045)$ & $16.431(015)$ & $15.727(015)$ & $15.565(015)$ & $16.979(015)$ & $16.014(015)$ & Site 3 \\
\hline 54179.49 & $19.420(081)$ & $16.858(015)$ & $16.005(015)$ & $15.741(015)$ & $17.492(015)$ & $16.354(015)$ & Site 3 \\
\hline 54185.50 & $19.842(157)$ & $17.147(015)$ & $16.246(015)$ & $15.981(015)$ & $17.816(015)$ & $16.623(015)$ & Site 3 \\
\hline 54190.49 & $19.740(213)$ & $17.278(015)$ & $16.404(015)$ & $16.127(015)$ & $17.915(021)$ & $16.726(015)$ & Site 3 \\
\hline 54194.48 & $19.418(146)$ & $17.384(015)$ & $16.514(015)$ & $16.231(015)$ & $17.956(026)$ & $16.845(015)$ & Site 3 \\
\hline 54197.48 & $19.478(103)$ & $17.429(015)$ & $\cdots$ & $\cdots$ & $18.063(015)$ & $\cdots$ & Site 3 \\
\hline 54204.47 & $19.399(152)$ & $\ldots$ & $\ldots$ & $\cdots$ & $\cdots$ & $17.034(015)$ & Site 3 \\
\hline 54204.48 & $19.408(104)$ & $\ldots$ & $\ldots$ & $\ldots$ & $\ldots$ & $\ldots$ & Site 3 \\
\hline 54434.11 & $\cdots$ & $\cdots$ & $20.951(077)$ & $20.658(108)$ & $22.517(127)$ & $21.900(081)$ & FORS1 \\
\hline 54507.03 & $\cdots$ & $\cdots$ & $22.151(162)$ & $21.983(150)$ & $23.521(185)$ & $23.159(125)$ & FORS1 \\
\hline
\end{tabular}

Notes. Bolometric maximum occured on JD-2454163.12. Uncertainties given in parentheses are in millimag. FORS1 imaging was conducted with a set of standard Johnson Kron-Cousins BVRI filters.

Table 3

CSP Near-Infrared Photometry of SN 2007Y

\begin{tabular}{|c|c|c|c|c|c|}
\hline $\mathrm{JD}-2,400,000$ & $Y$ & $J$ & $H$ & $K_{s}$ & Instrument \\
\hline 54150.51 & $16.679(036)$ & $16.628(044)$ & $16.618(063)$ & $\cdots$ & RetroCam \\
\hline 54156.53 & $15.631(015)$ & $15.628(014)$ & $\ldots$ & $\ldots$ & RetroCam \\
\hline 54158.54 & $15.416(012)$ & $15.397(012)$ & $15.341(031)$ & $15.187(044)$ & WIRC \\
\hline 54159.52 & $15.337(012)$ & $15.319(013)$ & $15.247(031)$ & $15.079(044)$ & WIRC \\
\hline 54162.50 & $15.109(015)$ & $15.063(013)$ & $14.975(028)$ & $\ldots$ & RetroCam \\
\hline 54164.50 & $14.995(013)$ & $14.946(015)$ & $14.864(025)$ & $\cdots$ & RetroCam \\
\hline 54167.51 & $14.935(012)$ & $14.830(011)$ & $14.748(022)$ & $\cdots$ & RetroCam \\
\hline 54168.52 & $14.962(013)$ & $14.845(014)$ & $14.744(023)$ & $\cdots$ & RetroCam \\
\hline 54169.52 & $14.969(016)$ & $14.835(009)$ & 14.751(029) & $\cdots$ & WIRC \\
\hline 54170.51 & $15.009(016)$ & $14.822(010)$ & $14.698(030)$ & $14.505(037)$ & WIRC \\
\hline 54171.50 & $15.031(016)$ & $14.888(008)$ & $14.743(029)$ & $14.481(036)$ & WIRC \\
\hline 54172.50 & $15.070(016)$ & 14.899(009) & $14.770(029)$ & $14.504(036)$ & WIRC \\
\hline 54175.51 & $15.125(014)$ & $15.030(019)$ & $14.745(031)$ & $\cdots$ & RetroCam \\
\hline 54189.50 & $15.276(021)$ & $15.521(027)$ & $15.124(049)$ & $\cdots$ & RetroCam \\
\hline 54507.06 & $\ldots$ & $>21.502$ & $>21.380$ & $>21.219$ & ISAAC \\
\hline
\end{tabular}

Notes. Bolometric maximum occured on JD-2454163.12. Uncertainties given in parentheses are in millimag.

been shifted below the top one by an arbitrary constant. The number in parentheses gives the epoch with respect to $L_{\max }$. Four of these spectra were taken when the ejecta was fully nebular. A subset of these are modeled in order to derive the physical parameters of SN 2007Y (see Section 3.7).

\section{ANALYSIS}

\subsection{Distance and Reddening}

With coordinates of $\alpha=03^{\mathrm{h}} 02^{\mathrm{m}} 35^{\mathrm{s}} .92$ and $\delta=-22^{\circ} 53^{\prime} 50^{\prime \prime} .1$, SN 2007 Y was located $24^{\prime \prime}$ west and $123^{\prime \prime}$ south of the nucleus of NGC 1187; well outside of any spiral arm. The NASA/IPAC Extragalactic Database (NED) lists a heliocentric recessional velocity for this galaxy of $1390 \mathrm{~km} \mathrm{~s}^{-1}$. With a value of $H_{\circ}=$ $72 \pm 8 \mathrm{~km} \mathrm{~s}^{-1} \mathrm{Mpc}^{-1}$ (Freedman et al. 2001) and adopting a $400 \mathrm{~km} \mathrm{~s}^{-1}$ uncertainty in the redshift distance, the distance to NGC 1187 is $19.31 \pm 5.56 \mathrm{Mpc}(\mu=31.43 \pm 0.55)$. If this distance is corrected for Virgo infall it becomes $10 \%$ smaller. In this paper, we will use the more traditional heliocentric distance, but note that a Virgo infall corrected distance would make our estimated values of the peak absolute magnitude, luminosity, and mass of ${ }^{56} \mathrm{Ni}$ about $10 \%$ less. 
Table 4

Spectroscopic Observations of SN 2007Y

\begin{tabular}{|c|c|c|c|c|c|c|}
\hline $\begin{array}{l}\text { Phase } \\
\text { JD-2, 400, } 000\end{array}$ & $\begin{array}{l}\text { Epoch }^{\mathrm{a}} \\
\text { (days) }\end{array}$ & Telescope & Instrument & $\begin{array}{c}\text { Range } \\
(\AA)\end{array}$ & $\begin{array}{l}\text { Resolution } \\
\text { (Å per pixel) }\end{array}$ & $\begin{array}{c}\text { Exposure } \\
\text { (s) }\end{array}$ \\
\hline 54150.54 & -14 & Du PONT & WFCCD & 3800-9235 & 3.0 & 600 \\
\hline 54156.52 & -8 & Du PONT & WFCCD & 3800-9235 & 3.0 & 600 \\
\hline 54163.57 & -1 & NTT & EMMI & $3200-10200$ & 3.3 & 200 \\
\hline 54170.50 & +6 & BAADE & IMACS & $3790-10300$ & 2.0 & 600 \\
\hline 54173.51 & +9 & Du PONT & $\mathrm{B} \& \mathrm{C}$ & $3430-9650$ & 3.0 & 600 \\
\hline 54178.50 & +14 & Du PONT & $\mathrm{B} \& \mathrm{C}$ & $3420-9630$ & 3.0 & 600 \\
\hline 54185.49 & +21 & Du PONT & $\mathrm{B} \& \mathrm{C}$ & 3400-9600 & 3.0 & 600 \\
\hline 54203.48 & +39 & Du PONT & WFCCD & 3800-9235 & 3.0 & 800 \\
\hline 54363.82 & +201 & NTT & EMMI & $3750-8160$ & 3.0 & 1800 \\
\hline 54394.86 & +230 & CLAY & LDSS3 & 4100-9980 & 1.2 & $900^{\mathrm{b}}$ \\
\hline 54421.78 & +257 & BAADE & IMACS & $3800-10675$ & 2.0 & 1800 \\
\hline 54434.57 & +270 & VLT & FORS1 & $3640-8880$ & 3.3 & 1280 \\
\hline
\end{tabular}

Notes. Some spectra are the combination of multiple observations.

${ }^{a}$ Days since $L_{\max }$

b Different exposure times in blue channel (900 s) and red channel (600 s).

According to the infrared dust maps of Schlegel et al. (1998), the reddening due to the Milky Way in the direction of NGC 1187 is $E(B-V)_{\text {gal }}=0.022$ mag. The optical colors of SN 2007Y near peak brightness (see below) are relatively blue, suggesting minimal host galaxy extinction. A spectrum obtained near maximum light shows the presence of a weak $\mathrm{Na}$ I $\mathrm{D}$ absorption feature at the redshift of the host galaxy with an equivalent width of $0.56 \AA$. Using the relationship between the equivalent width of the $\mathrm{Na}$ I D and $E(B-V)$ (see Turatto et al. 2003; Taubenberger et al. 2006) suggests a host galaxy reddening of $E(B-V)_{\text {host }}=0.09$ mag. Combining this with the Galactic reddening gives $E(B-V)_{\text {tot }}=0.112 \mathrm{mag}$. This value is adopted in the subsequent analysis.

\subsection{X-Ray Observations and Limiting Luminosity}

Swift X-Ray Telescope (XRT; Burrows et al. 2005) observations were obtained simultaneously with the UVOT observations. To search for X-ray emission from SN 2007Y, we extracted X-ray counts from a circular region with a 10 pixel radius (23:7, corresponding to the XRT on-axis $90 \%$ encircled energy radius) centered on the optical position of the SN. The background was extracted locally from a source-free region of radius of $40^{\prime \prime}$ to account for detector and sky background, and for diffuse emission from the host galaxy.

No X-ray source is detected in the merged $66.5 \mathrm{ks}$ XRT data obtained in photon-counting mode. The $3 \sigma$ upper limit to the XRT net count rate is $2.63 \times 10^{-4} \mathrm{cts} \mathrm{s}^{-1}$, corresponding to an unabsorbed $\left(0.2-10 \mathrm{keV}\right.$ band) X-ray flux of $f_{0.2-10}<$ $1.24 \times 10^{-14} \mathrm{erg} \mathrm{cm}^{-2} \mathrm{~s}^{-1}$ and a luminosity $L_{0.2-10}<5.5 \times$ $10^{38} \mathrm{erg} \mathrm{s}^{-1}$ for an adopted thermal plasma spectrum with a temperature of $k T=10 \mathrm{keV}$ (see Fransson et al. 1996 and references therein), a Galactic foreground column density of $N_{\mathrm{H}}=1.91 \times 10^{20} \mathrm{~cm}^{-2}$ (Dickey \& Lockman 1990) and a distance of $19.31 \mathrm{Mpc}$.

In order to search for a possible late X-ray turn-on phase, we binned the XRT data into two time intervals ranging from day -14 to +23 and day +290 to +387 with exposure times of $56.5 \mathrm{ks}$ and $10.1 \mathrm{ks}$, respectively. No X-ray source is found at the position of SN 2007Y in either of the two epochs, down to $3 \sigma$ limiting luminosities of $<6.5 \times 10^{38} \mathrm{erg} \mathrm{s}^{-1}$ and $<1.9 \times 10^{39} \mathrm{erg} \mathrm{s}^{-1}$, respectively.

The lack of X-ray emission can be used to put loose limits on the mass-loss rate of the progenitor system. Following the discussion in Immler et al. (2007, and references therein), an upper limit to the mass-loss rate of $\dot{M} \approx 2 \times 10^{-5} M_{\odot} \mathrm{yr}^{-1}$ $\left(v_{\mathrm{w}} / 10 \mathrm{~km} \mathrm{~s}^{-1}\right)$ with an uncertainty of a factor of $2-3$ is obtained. In this calculation the model assumes thermal emission, which is more appropriate for Type II SNe. In addition the wind velocity of Wolf-Rayet stars is known to be on the order of $1000 \mathrm{~km} \mathrm{~s}^{-1}$. Taking this into account then implies a limit on the mass-loss of $\sim 2 \times 10^{-3} M_{\odot} \mathrm{yr}^{-1}$. To obtain a more robust estimate on the mass-loss rate we turn to constraints provided by the radio observations (see Section 3.8).

\subsection{Ultraviolet, Optical, and Near-infrared Light Curves}

The nicely sampled ultraviolet, optical, near-infrared light curves presented in Figure 2 constitute one of the more complete collections obtained for a He-rich SN Ib.

Under close inspection the CSP and UVOT $B$ - and $V$-band light curves exhibit excellent agreement with one another. However, the $U$-band light curve of the UVOT is systematically $\sim 0.7$ mag brighter than CSP's $u^{\prime}$-band light curve. This is not surprising considering the appreciable difference between the transmission functions of these two filters. The $u^{\prime}$ band has an effective wavelength $\lambda_{\text {eff }}=3681 \AA$ and a FWHM of $487 \AA$, while the $U$ band has $\lambda_{\text {eff }}=3465 \AA$ and a FWHM of $785 \AA$.

To see if this discrepancy could be attributed to the differences in filter transmissivities we performed an experiment using spectrophotometry of SN 2007Y. First, appropriate zero points were computed for each passband using a library of spectrophotometric flux standards (Stritzinger et al. 2005). Armed with these zero points and the early phase spectra that had their wavelength coverage extended to the atmospheric cutoff by assuming zero flux, synthetic magnitudes of SN $2007 \mathrm{Y}$ were determined for the two passbands. We find the $U$-band filter consistently gives synthetic magnitudes brighter than $u^{\prime}$-band, and the disparity is in agreement with the average difference between the broadband magnitudes.

Figure 5 shows the $\left(u^{\prime}-B\right)$ and $(B-V)$ color curves of SN 2007Y, both of which track photospheric temperature variations. Included for comparison are the color curves of two well observed SNe IIb: SN 2008ax (Pastorello et al. 2008) and SN 2008aq (CSP paper in preparation). Also included is the ( $B$ $-V$ ) color curve of the Type Ib SN 1999ex (Stritzinger et al. 2002). The color curves of each $\mathrm{SNe}$ have been corrected for extinction using reddening values of $E(B-V)$ of $0.30,0.11$, 


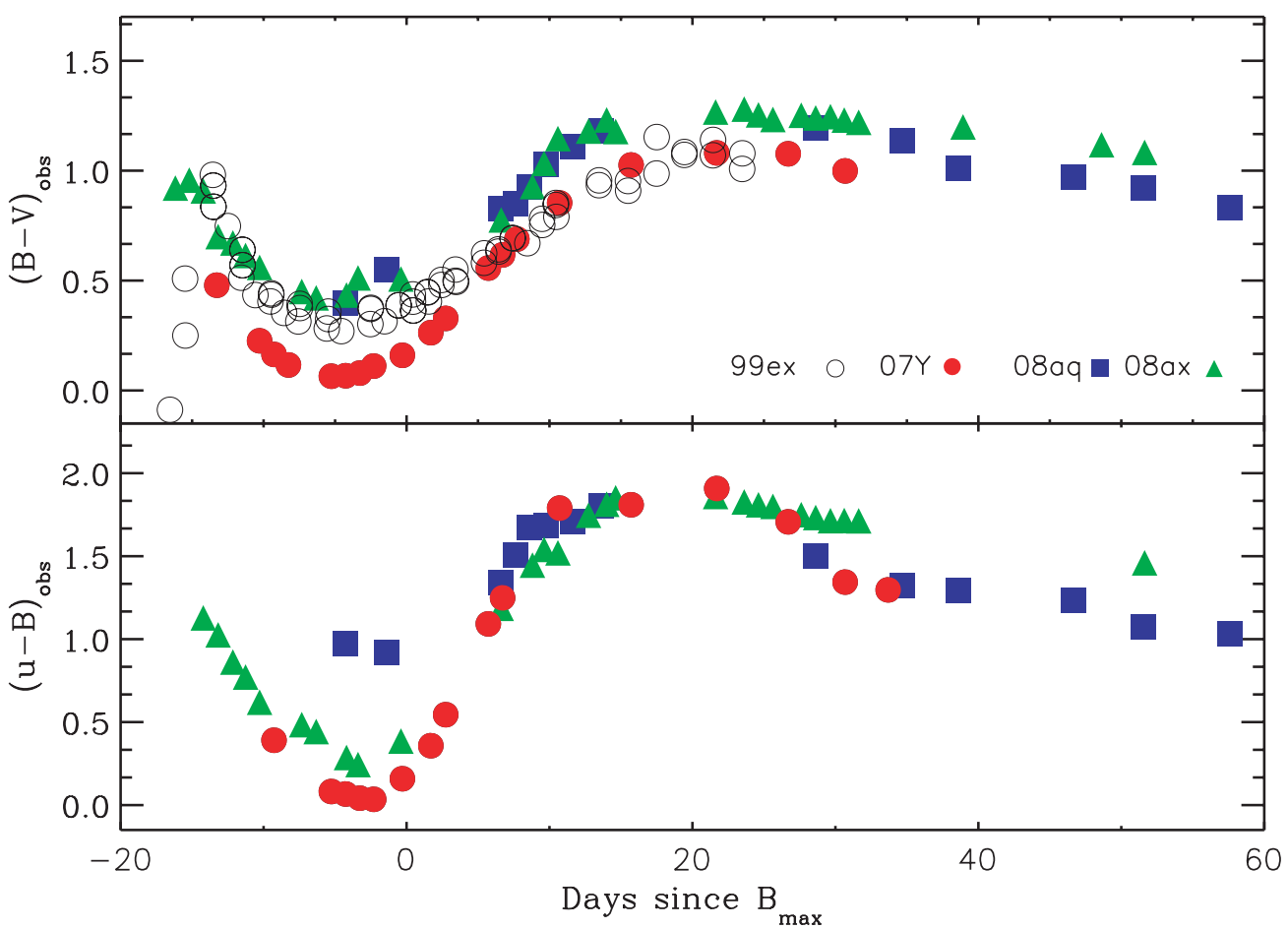

Figure 5. $\left(u^{\prime}-B\right)_{\mathrm{obs}}$ and $(B-V)_{\mathrm{obs}}$ color curves of SN 2007Y (red filled circles), SN 2008ax (green triangles), SN 2008aq (blue squares), and the ( $\left.B-V\right)_{\mathrm{obs}}$ color curve of SN 1999ex (black circles).

(A color version of this figure is available in the online journal.)

0.05, and 0.30 for SN 1999ex, SN 2007Y, SN 2008aq and SN 2008ax, respectively. As the brightness of the SN increases the photospheric temperature also increases, which in turn, causes the color curves to evolve to the blue. Specifically, the color of SN 2007Y decreases from a value of $(B-V)=0.47$ on day -12.8 to $(B-V)=0.06$ on day -4.3 . The colors then evolve monotonically back to the red where $(B-V)=1.0$ on day +31.2 . Then the colors moved marginally back toward the blue. Interestingly, around 3 weeks past maximum the UVOT ultraviolet light curves appear to stop declining in brightness. Although UVOT observations were discontinued near this epoch the $u^{\prime}$-band light curve indicates that the flux in this passband not only stopped declining but actually increased. Specifically, the $u^{\prime}$ band decreases from $19.800 \mathrm{mag}$ on day +22 to $19.408 \pm$ 0.11019 days later. This phenomenon coincides with a $\left(u^{\prime}-B\right)$ color evolution to the blue that is more prevalent than in other He-rich core-collapse SN like SN 2008ax (see the bottom panel of Figure 5). A blue excess is also seen in SN 2008aq where the $u^{\prime}$-band light curve becomes brighter by $0.63 \mathrm{mag}$ from day +30 to +96 . This is followed by a dimming of the $u^{\prime}$ light curve by $0.55 \mathrm{mag}$ from day +96 to +123 . Note in the bottom panel of Figure 5 the $\left(u^{\prime}-B\right)$ colors curves of SN 2007Y and SN 2008aq are very similar between days +25 and +35 .

The excess in ultraviolet emission could be connected to a change in the opacity of the ejecta. Unfortunately the spectra do not extend far enough in the blue to determine if the spectral energy distribution of the SN actually increases in the region covered by the $u^{\prime}$-band filter. Alternatively, this could be the consequence of shock heating produced from the interaction of high-velocity SN ejecta with CSM (e.g., Immler et al. 2006). As we will see below, there is evidence in the late epoch optical spectrum of such an interaction.

We now turn to the broadband absolute magnitudes of SN 2007Y. First the time and observed magnitude at maximum light
Table 5

Lightcurve Parameters

\begin{tabular}{lccc}
\hline \hline Filter & $\begin{array}{c}\text { Peak Time } \\
(\mathrm{JD}-2,400,000)\end{array}$ & $\begin{array}{c}\text { Peak Obs. } \\
(\mathrm{Mag})\end{array}$ & $\begin{array}{c}\text { Peak Abs. } \\
(\mathrm{Mag})\end{array}$ \\
\hline$w 2$ & 54161.3 & $18.348(050)$ & $-15.236(578)$ \\
$m 2$ & 54162.5 & $19.394(200)$ & $-15.351(616)$ \\
$w 1$ & 54161.4 & $16.903(050)$ & $-17.303(578)$ \\
$U$ & 54161.4 & $15.057(010)$ & $-16.951(578)$ \\
$u^{\prime}$ & 54161.6 & $15.849(013)$ & $-16.129(576)$ \\
$B$ & 54163.3 & $15.647(012)$ & $-16.242(576)$ \\
$g^{\prime}$ & 54163.8 & $15.437(006)$ & $-16.396(576)$ \\
$V$ & 54165.6 & $15.336(011)$ & $-16.446(576)$ \\
$r^{\prime}$ & 54166.5 & $15.292(010)$ & $-16.430(576)$ \\
$i^{\prime}$ & 54166.9 & $15.326(010)$ & $-16.325(576)$ \\
$Y$ & 54167.2 & $14.948(031)$ & $-16.597(579)$ \\
$J$ & 54169.2 & $14.839(028)$ & $-16.686(577)$ \\
$H$ & 54171.4 & $14.733(069)$ & $-16.757(581)$ \\
$K_{s}$ & 54171.4 & $14.494(640)$ & $-16.975(985)$ \\
\hline
\end{tabular}

Notes. Bolometric maximum occured on JD-2454163.12. Uncertainty in time of peak magnitude is 0.5 days for each filter except the $m 2$ - and $K_{s}$-bands. Their estimated time of maximum has a 2 day uncertainty.

of each filter light curve was estimated with the use of moderateorder (5-8) polynomial fits; these values are given in Table 5. Similarly to other $\mathrm{SNe} \mathrm{Ib} / \mathrm{c}$, the ultraviolet/blue light curves of SN 2007 Y peak $\sim 4$ days prior to the optical light curves. This is followed by the near-infrared bands peaking 3-5 days later. Peak absolute magnitudes were computed using the reddening value of $E(B-V)_{\text {tot }}=0.112 \mathrm{mag}$, an $R_{V}$ value of 3.1 , and a distance of $19.31 \pm 5.56 \mathrm{Mpc}$ (see Section 3.1). These values are also listed in Table 5 with the quoted errors accounting for uncertainty in the estimated value of peak magnitude and the distance to the host galaxy. With an absolute magnitude of 

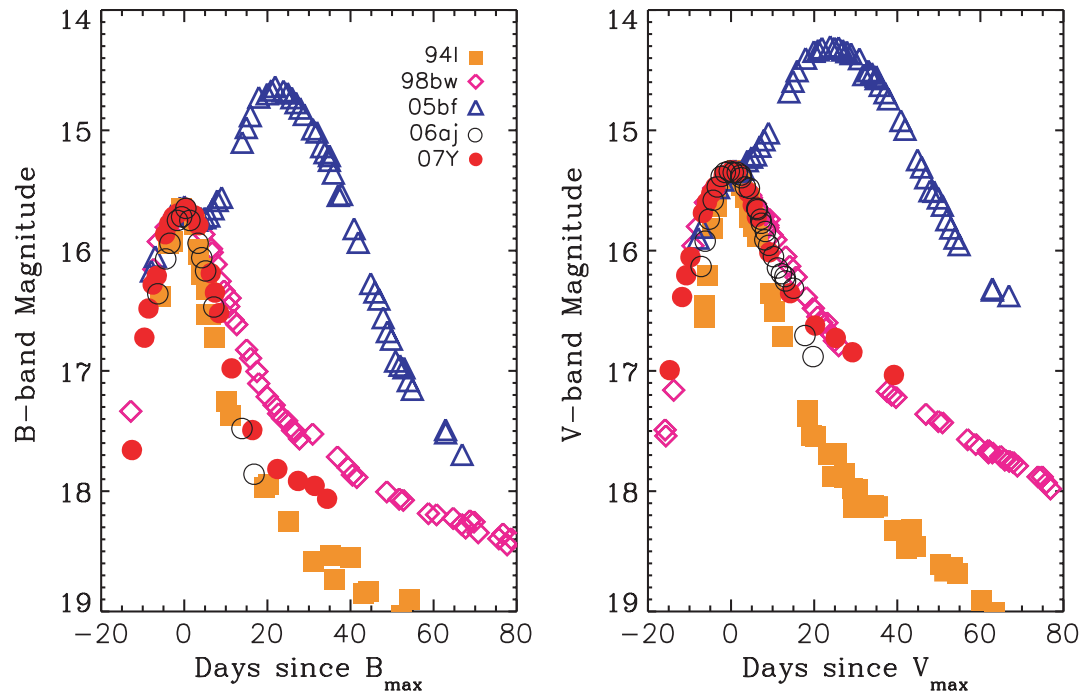

Figure 6. Comparison of the $B$ - and $V$-band light curves of SN 2007Y to those of SNe 1994I, 1998bw, 2005bf and 2006aj. The light curves of the comparison SNe $\mathrm{Ib} / \mathrm{c}$ have been shifted to match the time and value of $L_{\max }$ for SN 2007Y. Light curves of SNe 1998bw and 2006aj have been corrected for time dilation. Photometry of SN 2006aj has had the host galaxy contribution removed. SN 2007Y rises similar to SN 1998bw, however, after maximum it evolves significantly faster. After $\sim 2$ weeks past maximum the rate of decline in SN 2007Y weakened causing the $V$ band to match again SN 1998bw. The first peak in SN 2005bf has a similar rise compared to SN 2007Y, but then evolves to another brighter secondary peak.

(A color version of this figure is available in the online journal.)

$M_{V}=-16.5 \pm 0.6 \mathrm{mag}, \mathrm{SN} 2007 \mathrm{Y}$ is similar in brightness to SN 2008D, which peaked at $M_{V} \approx-16.7$ mag (Soderberg et al. 2008). This turns out to be on the faint end of the absolute magnitude distribution of SNe Ib/c. For example, SN 2006aj peaked at $M_{V}=-18.7 \pm 0.1$ (Modjaz et al. 2006), while other previously observed GRB/X-ray transient related $\mathrm{SNe} \mathrm{Ib} / \mathrm{c}$ have peaked at $M_{V}<-20.0$ mag (see Richardson et al. 2006 and references therein).

In Figure 6 the $B$ - and $V$-band light curves of SN 2007Y are compared with those of four other well observed $\mathrm{SNe} \mathrm{Ib} / \mathrm{c}$ : SN 1994I (Richmond et al. 1996), SN 1998bw (Galama et al. 1998), SN 2005bf (Folatelli et al. 2006), and SN 2006aj (Mirabal et al. 2006; Sollerman et al. 2006). The observed magnitudes of the comparison $\mathrm{SNe} \mathrm{Ib} / \mathrm{c}$ have been normalized to the peak magnitudes of SN 2007Y and shifted in time to their respective epoch of peak brightness. The light curves of SN 1998bw and SN 2006aj have been corrected for time dilation, and SN 2006aj has also had its underlying host galaxy contribution removed. SN 2007Y rises to maximum like SN 1998bw and SN 2005bf; however, after maximum it declines rapidly like SN 2006aj. Then about three weeks past maximum the rate of decline begins to slow, and again, the $V$-band light curve of SN $2007 \mathrm{Y}$ is similar to that of SN 1998bw. We note that SN 1999ex and SN 2008D are not included in Figure 6, but if they were, one would see that the width of their light curves is significantly broader than SN 2007Y. Overall the shape of the $V$-band light curve, which contains the majority of flux around maximum, is most similar to SN 2006aj.

Looking at Arnett (1982)'s analytical description of the shape of the light curve around peak brightness, we can infer from the similar shaped light curves that the effective diffusion time $\tau_{\mathrm{m}}$, which can expressed as

$$
\tau_{\mathrm{m}} \propto \kappa_{\mathrm{opt}}^{1 / 2} M_{\mathrm{ejc}}^{3 / 4} E_{\mathrm{kin}}^{-1 / 4},
$$

is similar in SNe 2006aj and 2007Y. In this expression $\kappa_{\text {opt }}$ refers to the mean optical opacity, $M_{\mathrm{ejc}}$ is the ejecta mass, and $E_{\text {kin }}$ is the kinetic energy of the expansion. This then implies the ratio of $M_{\mathrm{ejc}}^{3}$ to $E_{\mathrm{kin}}$ is also similar between these two events.
Now we turn our attention to the late phase VLT photometry shown in Figure 3. The optical light curves indicate magnitude differences from peak to the last observed epoch of $\Delta m(B)=$ 7.9 and $\Delta m(R)=6.9$. Furthermore, a weighted least-squares analysis gives decline rates per hundred days of $B=1.30 \pm$ $0.31, V=1.73 \pm 0.20, R=1.65 \pm 0.25$ and $I=1.82 \pm 0.25$. Clearly these decline rates are faster than the decline of 0.98 mag per hundred days that is expected in the case of complete trapping of the $\gamma$ rays produced from the decay of ${ }^{56} \mathrm{Co}$ to ${ }^{56} \mathrm{Fe}$. Although the measured magnitude decline rates are rather uncertain given that only two epochs were observed, they are nonetheless in agreement with other $\mathrm{SNe} \mathrm{Ib} / \mathrm{c}$ (e.g., Barbon et al. 1994; Sollerman et al. 1998; Patat et al. 2001; Elmhamdi et al. 2004; Tomita et al. 2006; Clocchiatti et al. 2008), and hints toward a low ejecta mass.

\subsection{Bolometric Light Curve}

The comprehensive wavelength coverage provided by the early-time observations affords an excellent opportunity to construct a nearly complete bolometric light curve of SN 2007Y. To this end it was first necessary to account for gaps in any individual light curve when a particular passband was not observed on a given night. In these cases, it proved necessary to interpolate between the existing light curve using low order polynomial fits. As the $m 2$-band light curve covers only a limited time around maximum its temporal coverage was extended by extrapolation assuming a color correction (based on the available photometry) of $(w 2-m 2)=-0.929$. Similarly, as the $K_{s}$ light curve sampling was rather sparse a $\left(V-K_{S}\right)=1.0$ color was adopted to extend the light curve during the premaximum phase, and a $\left(V-K_{s}\right)=0.2$ color was adopted to extrapolate the light curve from day +12 to +42 . Although these two assumptions are rather simple they do provide a reasonably accurate extrapolation of the light curves.

Next, the photometry was corrected for extinction using our adopted value of reddening and a $R_{V}=3.1$ extinction curve for the optical and near-infrared data, while the extinction curve of Pei (1992) was used to correct the ultraviolet photometry. 


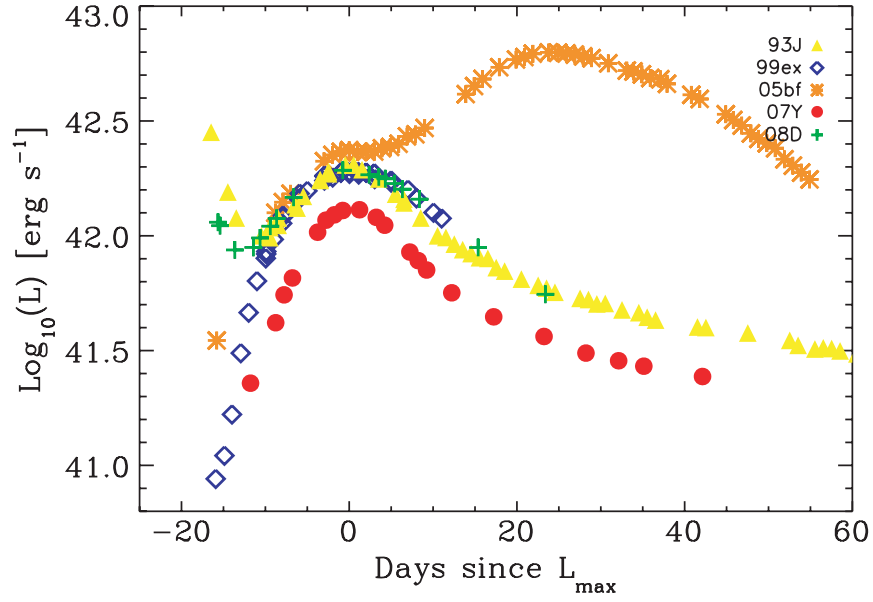

Figure 7. Comparison of the bolometric (UVOIR) light curves of SN 2007Y (red filled circles) with the SNe Ib 1999ex (blue diamonds), 2005bf (orange stars), 2008D (green crosses), and the SN IIb 1993J (yellow triangles). A reddening of $E(B-V)=0.112 \mathrm{mag}$ and a distance of $19.31 \mathrm{Mpc}$ have been adopted to place the UVOIR light curve of SN 2007Y on an absolute flux scale. Note that the last four epochs of SN 2007Y do not include any contribution from the ultraviolet.

(A color version of this figure is available in the online journal.)

The magnitudes were then converted to flux at the effective wavelength of each filter. In the case of the UVOT photometry, zero points and count-rate-to-flux conversion factors given in Tables 6 and 9 of Poole et al. (2008) were used.

To compute the quasi-bolometric (UV to NIR, hereafter UVOIR) light curve the observed magnitudes of each light curve were converted to flux and then integrated over frequency. The summed flux was then converted to luminosity using our adopted distance to NGC 1187 . Note the last four epochs of the early phase UVOIR light curve were computed without ultraviolet observations, and the late phase bolometric fluxes were derived with $B V R I J_{s} H$ photometry. In Figure 7 the UVOIR light curve of SN 2007Y is compared to the UVOIR light curves of several low luminosity SNe Ib. These include: SN 1999ex (Stritzinger et al. 2002), SN 2005bf (Folatelli et al. 2006), SN 2008D (Malesani et al. 2009), and the Type IIb SN 1993J (Richmond et al. 1994). Extinction values of $A_{V}=0.6,0.93$, and $2.5 \mathrm{mag}$, and distances of 3.6, 44.1, and $30.0 \mathrm{Mpc}$ were adopted for SN 1993J, SN 1999 ex and SN 2008D, respectively. In the case of SN 2005bf, values of ' $L_{\mathrm{bol}}$ ' were taken directly from Table 2 of Folatelli et al. (2006).

The UVOIR light curve of SN 2007Y indicates a peak luminosity of $\sim 1.30 \times 10^{42} \mathrm{erg} \mathrm{s}^{-1}$. The main uncertainty in this estimate is the error in the distance which is on the order of $30 \%$. One way to estimate the abundance of ${ }^{56} \mathrm{Ni}$, the main radioactive isotope that powers the light curves of $\mathrm{SNe}$, is through the use of Arnett's rule (Arnett 1982). Although based on a number of underlying assumptions, Richardson et al. (2006) have used this method to obtain reasonable estimates of the ${ }^{56} \mathrm{Ni}$ yields for a sample of two dozen SNe Ib/c. In the case of SN 2007Y, if we assume a rise time of 18 days, application of Arnett's rule tells us $\approx 0.06 \pm 0.02 M_{\odot}$ of ${ }^{56} \mathrm{Ni}$ was synthesized in SN 2007Y. ${ }^{21}$

An alternative method of estimating the ${ }^{56} \mathrm{Ni}$ yield is to fit a radioactive decay energy deposition function to the late phase UVOIR light curve. Under the reasonable assumptions that the

\footnotetext{
${ }^{21}$ We note in passing that the rise times of SNe Ib vary significantly. The rise times of SNe 1999ex and 2008D were 18 days (Stritzinger et al. 2002; Modjaz et al. 2009), while SN 2006aj exhibited a rise time of 11 days (Sollerman et al. 2006)
}

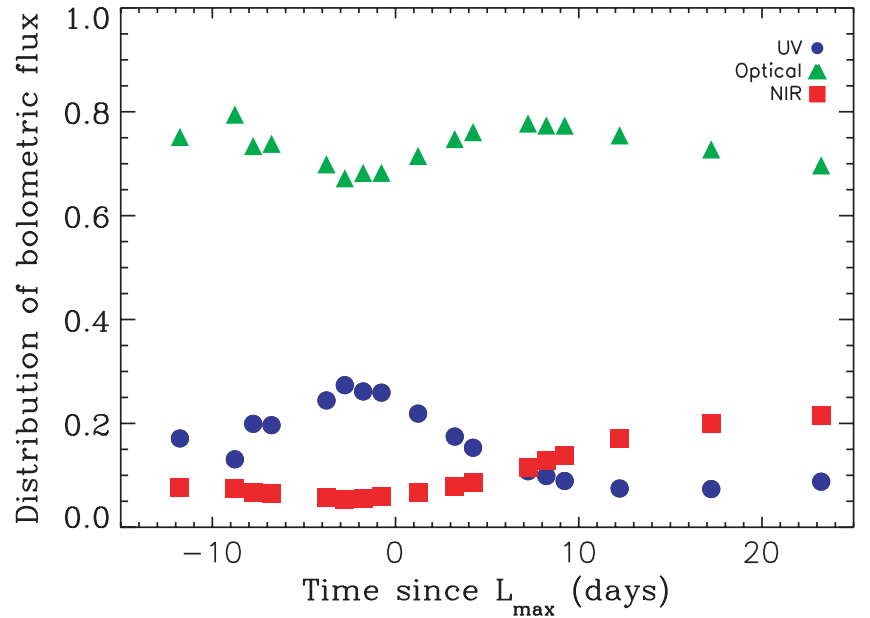

Figure 8. Distribution of flux as a function of time in three wavelength regimes. Filled blue circles represent the flux blueward of the atmospheric cutoff ( $3150 \AA$ ), filled green triangles the optical flux, and filled red squares the near-infrared $(\sim 0.95$ to $2.5 \mu)$ contribution.

(A color version of this figure is available in the online journal.)

majority of energy deposited in the ejecta at late phases is from the ${ }^{56} \mathrm{Co} \rightarrow{ }^{56} \mathrm{Fe}$ decay, and that the optical depth, $\tau$, is much less than one, a simple model that estimates the ${ }^{56} \mathrm{Ni}$-mass can be fitted to the UVOIR light curve from 50 to 350 days. The functional form of the model used (see Sollerman et al. 1998) is $L=1.3 \times 10^{43} M_{\mathrm{Ni}} e^{-t / 111.3}\left(1-0.966 e^{-\tau}\right) \mathrm{ergs}^{-1}$, where $\tau$ is given by $\left(t_{1} / t\right)^{2}$. In this form $t_{1}$ sets the time when the optical depth to $\gamma$ rays is unity.

Before the energy deposition function could be used to estimate the ${ }^{56} \mathrm{Ni}$ mass it first deemed necessary to extend the UVOIR light curve from day +41 to +60 . This was achieved by applying a linear least-squares fit to the UVOIR light curve from day +28 to +41 ; this best fit was then used to extrapolate the light curve to day +60 . Note the assumption that the decline rate between days +28 to +41 can be used to extend all but the $u^{\prime}$-band light curves out to day +60 is reasonable as other $\mathrm{SNe} \mathrm{Ib} / \mathrm{c}$ show no changes in decline rate during the epochs in question (see Clocchiatti et al. 2008). Finally the best fit of the toy model to the UVOIR light curve was obtained with a $t_{1}$ of 35.8 days and a ${ }^{56} \mathrm{Ni}$ mass of $0.06 M_{\odot}$.

The broad wavelength coverage obtained for SN 2007Y also allows for an estimation of the distribution of flux (as a function of time) in the different wavelength domains. These estimates are shown in Figure 8. Similar to Type Ia $\mathrm{SNe}$ at maximum the majority of flux is emitted in the optical; however, the ultraviolet light curves contained a significant portion of flux $(\gtrsim 20 \%)$, while the near-infrared contribution was small, $\sim 5 \%$. By two weeks past $L_{\max }$ the flux in the near-infrared passbands increased to roughly $20 \%$ while in the ultraviolet it dropped to $\leqslant 10 \%$. During the last two epochs (day +270 and +344$) \sim 40 \%$ of the observed flux was contained in the $R$ and $I$ bands because these passbands coincide with the strong emission lines of $\mathrm{O}$ I, $\mathrm{H} \alpha, \mathrm{Fe}$ II, and $\mathrm{Ca}$ II that dominate the spectrum (see below). By day $+270 \sim 5 \%$ of the flux was contained in the $H$ band, while the near-infrared as a whole contributes no more than $15 \%$. Evidently at this phase near-infrared emission lines were not a major coolant.

\subsection{Early Phase Spectroscopic Evolution}

The early spectra of SN 2007Y (Figure 4) consist of a series of P-Cygni features superimposed on a blue pseudo continuum. 


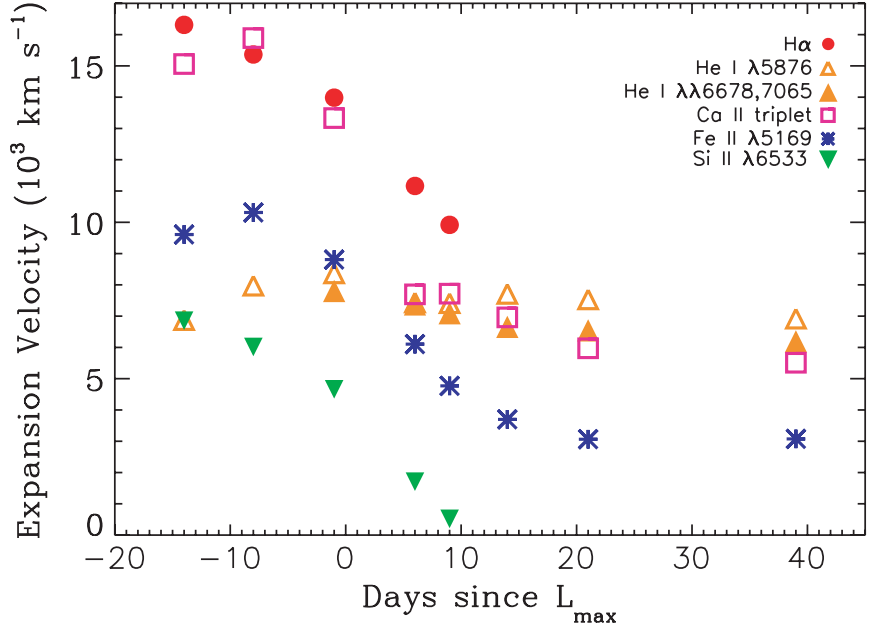

Figure 9. Velocity at maximum absorption of prominent ions in the spectra of SN 2007Y.

(A color version of this figure is available in the online journal.)

Multiple absorption and emission features of Fe II dominate the blue-end of the spectra while an exceptionally strong Ca II $\lambda \lambda 8498,8542,8662$ triplet rounds out the red-end. Distinctive features include: (1) an absorption trough between 4000 and $4200 \AA$ caused by the blending of Fe-group emission lines (i.e., Sc II, Ti II or alternatively Fe II); (2) absorption at $\sim 5900 \AA$ caused by a blend of the $\mathrm{Na}$ I $\lambda \lambda 5890,5896$ doublet with He I $\lambda 5876$; (3) a distinctive absorption feature at $\sim 6200 \AA$ that is possibly formed from a blending of $\mathrm{Si}$ II with high-velocity $\mathrm{H} \alpha$, and (4) a relatively weak $\mathrm{O}$ I $\lambda 7774$ absorption feature.

As SN 2007Y evolved through maximum, the strength of the absorption at $6200 \AA$ steadily declined until it disappeared around 10 days past $L_{\max }$. Meanwhile the depth of the Ca II triplet also decreased until day +6 when it transformed back to a deep absorption. This phenomenon reflects the transition of the $\mathrm{Ca}$ II triplet being formed from a high-velocity component to a photospheric component (see Figure 10; Folatelli et al. 2006; Parrent et al. 2007). After maximum, conspicuous lines of He I $\lambda \lambda 4471,4921,5016,6678,7065,7281$ emerge and begin to dominate the spectrum.

Figure 9 displays the time evolution of the blueshifts $\left(v_{\exp }\right)$ for the ions that produced the dominant features in the optical spectrum of SN 2007Y. The expansion velocity of Fe II $\lambda 6159$ is often used as an indicator of the photospheric velocity (Branch et al. 2002; Richardson et al. 2006). In the earliest spectra the absorption minimum of this line indicates that $v_{\text {exp }} \sim 10,000 \mathrm{~km}$ $\mathrm{s}^{-1}$, while $\mathrm{He} \mathrm{I}$ is measured as $v_{\exp } \sim 7000 \mathrm{~km} \mathrm{~s}^{-1}$. This is reminiscent of SN 2005bf, which to date has been the only other $\mathrm{SN} \mathrm{Ib}$ to show Fe II absorption blueshifted more than its $\mathrm{He}$ I absorption. As SN 2007Y evolved in time, Fe II $\lambda 5169$ monotonically declined, reaching a minimum value of $\sim 4500$ $\mathrm{km} \mathrm{s}^{-1}$ three weeks past maximum. On the other hand, the blueshift of He I $\lambda 5876$ shows an increase of $\sim 1500 \mathrm{~km} \mathrm{~s}^{-1}$ from day -14 to $L_{\max }$. Thereafter the blueshift of $\mathrm{He}$ I $\lambda 5876$ evolved from 8500 to $6500 \mathrm{~km} \mathrm{~s}^{-1}$ over the next month.

In contrast to all other ions, the velocity at maximum absorption of the $6200 \AA$ feature (assuming it is related to $\mathrm{H} \alpha$, see Section 4) and the Ca II triplet, suggest these features were produced from a component of gas that was detached from the photosphere with $v_{\exp } \sim 15,500 \mathrm{~km} \mathrm{~s}^{-1}$. By a week past maximum $\mathrm{H} \alpha$ had decreased to $v_{\exp } \sim 10,000 \mathrm{~km} \mathrm{~s}^{-1}$, while the

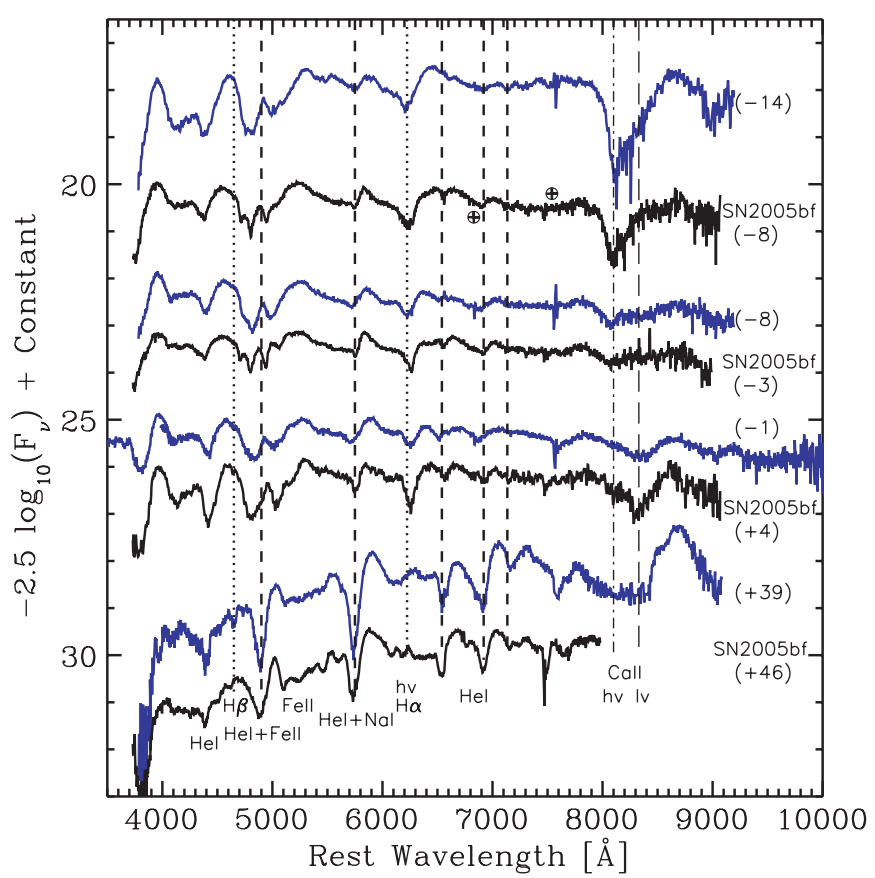

Figure 10. Comparison of similar epoch spectra of SN 2007Y (blue) and SN 2005bf (black; Folatelli et al. 2006). Labels in parentheses to the right of each spectrum indicated days from $L_{\max }$. In the case of SN 2005bf the time is with respect to its first maximum. The spectra are remarkably similar and have nearly an identical evolution. Ions responsible for some of the main features are indicated. Normal dash vertical line corresponds to He I $\lambda \lambda 4472,5876$, 6678, 7065, 7181; dotted lines are $\mathrm{H} \alpha$ and $\mathrm{H} \beta$, and high- and low-velocity components of $\mathrm{Ca}$ II are represented by dot-dashed and long dashed lines, respectively. Telluric features are indicated with an Earth symbol. Note that the first spectrum of SN 2007Y has been smoothed by averaging over 3 pixels.

(A color version of this figure is available in the online journal.)

Ca II triplet began to follow the velocity evolution of $\mathrm{He}$ I with $v_{\exp } \sim 6500 \mathrm{~km} \mathrm{~s}^{-1}$. The abnormally low blueshifts of Si II argues against the case that it is the dominate ion responsible for the formation of the $6200 \AA$ feature.

In Figure 10 spectra of SN 2007Y at days $-14,-8,-1$, and +39 are compared to spectra of SN 2005 bf obtained on days -8 , $-3,+4$, and $+42 .{ }^{22}$ The similarity between the earliest spectra of these two SNe Ib is remarkable. Two subtle differences exist, namely a somewhat stronger Ca II triplet in SN 2007Y, while the Fe II multiplet $42 \lambda \lambda 4924,5018,5169$ features in SN 2005bf are more narrow. In this figure, one can see the position of maximum absorption of the $\mathrm{Ca}$ II triplet clearly evolves in time. The narrow features in SN 2005bf, on the other hand, have been interpreted to be from a high-velocity component of $\mathrm{Fe}$ gas in the ejecta (Anupama et al. 2005; Folatelli et al. 2006; Parrent et al. 2007), which may be related to a polar outflow or a failed jet (Folatelli et al. 2006; Maeda et al. 2007b; Maund et al. 2007). The lack of these narrow features in SN 2007Y suggests its ejecta was more spherical than in the case of SN 2005bf.

Finally, at the bottom of Figure 10, spectra of SNe 2007Y and 2005bf taken over a month past maximum are compared. By this time the spectra of both events resemble normal $\mathrm{SNe} \mathrm{Ib}$.

\subsection{Nebular Phase Spectroscopy}

Months later, as SN 2007Y continued to expand, the photosphere receded deep into the ejecta leading to the onset of

\footnotetext{
${ }^{22}$ Epochs of SN 2005bf spectra are with respect to the time of its first
} maximum. 


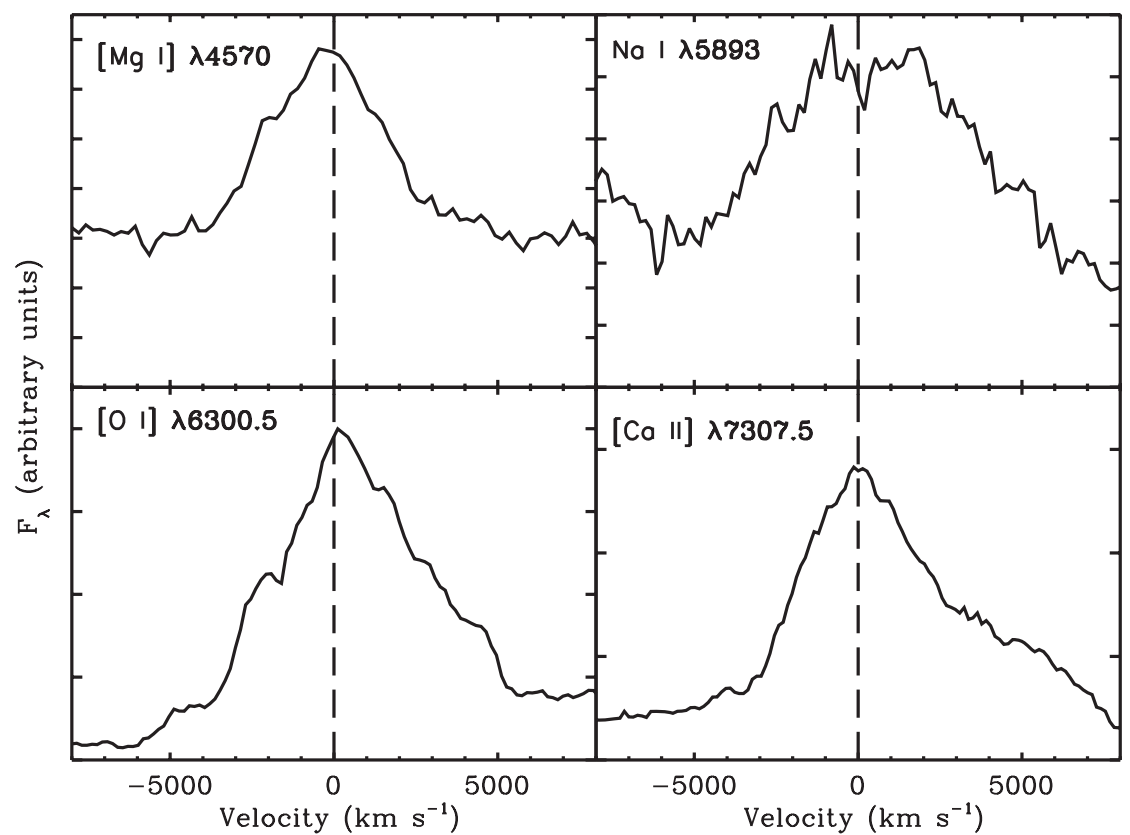

Figure 11. Prominent emission lines, in velocity space, from the day +270 nebular spectrum. The rest wavelength of each line is marked by a vertical dashed line. No major asymmetries or blueshifts are present.

the nebular phase. As radioactive heating and the formation of the nebular spectrum occurs at this time in what was the central region of the progenitor, the analysis and spectral synthesis of the late phase spectrum provide an excellent tool to estimate the abundances of stable and radioactive elements synthesized during the explosion. Furthermore, the study of line profiles and their relative strengths can provide clues regarding the geometry of the ejecta and the nature of the progenitor system.

The four nebular spectra at the bottom of Figure 4 exhibit many of the features typically associated with normal $\mathrm{SNe} \mathrm{Ib} / \mathrm{c}$. Strong emission lines from forbidden transitions of [O $\mathrm{I}]$ $\lambda \lambda 6300,6363$ and $[\mathrm{Ca}$ II] $\lambda \lambda 7291,7324$ clearly dominate the spectrum. The [Ca II] emission is actually blended with [Fe II] $\lambda \lambda 7155,7172,7388,7452$ and possibly $[\mathrm{O}$ II] $\lambda 7300$. Other less pronounced features identified include: $\mathrm{Mg}$ I] $\lambda 4570$, the $\mathrm{Na}$ I $\lambda \lambda 5890,5896$ doublet, the $\mathrm{Ca}$ II triplet, and a feature at $5200 \AA$ that is formed by a blending of [Fe II] lines. A marginal detection of $\mathrm{O}$ I $\lambda 7774$ is discernable, while the identification of $\mathrm{O}$ I $\lambda 5577$ is weak to nonexistent. Gaussian fits to the emission profiles indicate full-width-half-maximum (FWHM) velocities between 5000 to $7500 \mathrm{~km} \mathrm{~s}^{-1}$.

Plotted in Figure 11 are the emission line profiles, in velocity space, of Mg I], Na I, [O I] $\lambda 6300.5$, and [Ca II] $\lambda 7307.5$. Clearly these profiles show, in velocity space, no strong deviations from their rest wavelength, and all but $\mathrm{Na}$ I have a single peaked profile. The Na I profile, on the other hand, exhibits a moderately flat-topped profile. The shape and velocities of these line profiles can provide some insight into the distribution of the emitting material (Fransson \& Chevalier 1987), and the geometry of the explosion (Maeda et al. 2008). As the emission lines are observed to have similar widths, and that the profiles are symmetric and not sharply peaked, we conclude that there are no major asymmetries in the ejecta.

The most striking characteristic in the nebular spectra of SN $2007 \mathrm{Y}$ is a box-like emission profile red-ward of the [O I] $\lambda \lambda 6300,6363$ doublet. Superposed on this broad component are several narrow emission lines close to the expected position of $\mathrm{H} \alpha$. We identify these emission features as $\mathrm{H} \alpha$, [N II] $\lambda 6584$ and $[\mathrm{S}$ II] $\lambda 6717$, which are associated with an $\mathrm{H}$ II region in the vicinity of SN 2007Y. We measure a FWHM velocity of the narrow $\mathrm{H} \alpha$ component of $\leqslant 450 \mathrm{~km} \mathrm{~s}^{-1}$. The broad component, on the other hand, exhibits a velocity for the bulk of material to be located between 9000 and $11,000 \mathrm{~km} \mathrm{~s}^{-1}$. This material is undoubtedly hydrogen gas ejected from SN 2007Y.

Although rarely seen in other $\mathrm{SNe} \mathrm{Ib}$, a similar feature was also present in the Type Ib SN 1996N (Sollerman et al. 1998), and more notably in the well studied transitional Type IIb SN 1993J (Clocchiatti \& Wheeler 1994; Filippenko et al. 1994; Patat et al. 1995; Matheson et al. 2000). A similar feature was also reported in nebular spectra of SN 2005bf (Soderberg et al. 2005; Maeda et al. 2007b). Figure 12 shows a comparison of SN 2007Y (day +230) to SN 1993J (day +236) and SN 1996N $($ day +221$)$. Despite differences in profile shapes and blueshifts, these spectra are quite similar overall. During this epoch the $\mathrm{H} \alpha$ shoulder seems to be relatively stronger in SN 2007Y. In the case of SN 1993J the strength of this broad emission feature relative to the $\left[\mathrm{O}_{\mathrm{I}}\right]$ doublet continued to evolve in time until $\sim 500$ days after the explosion. By this epoch the ratio of the strengths of these two features reached unity (Matheson et al. 2000).

The source feeding the majority of emission in this broad wing is not radioactive heating, but rather, high-velocity hydrogen gas interacting with dense CSM (see Houck \& Fransson 1996 and Chevalier \& Fransson 1994 for details on the physics of this emission process). The CSM consists of mass shed by the progenitor, while the high-velocity hydrogen gas came from a thin shell of material ejected by the SN itself. The velocity of the hydrogen gas measured from the nebular spectrum is consistent with that derived for $\mathrm{H} \alpha$ from the $6200 \AA$ absorption feature nine days past maximum.

Interestingly, as is evident from Figure 12, the strength of [Ca II] $\lambda 7307.5$ relative to the [O I] $\lambda 6300$ doublet is comparable for all three SNe. Specifically, the $[\mathrm{Ca}$ II $] /[\mathrm{O}$ I] ratio of the three 


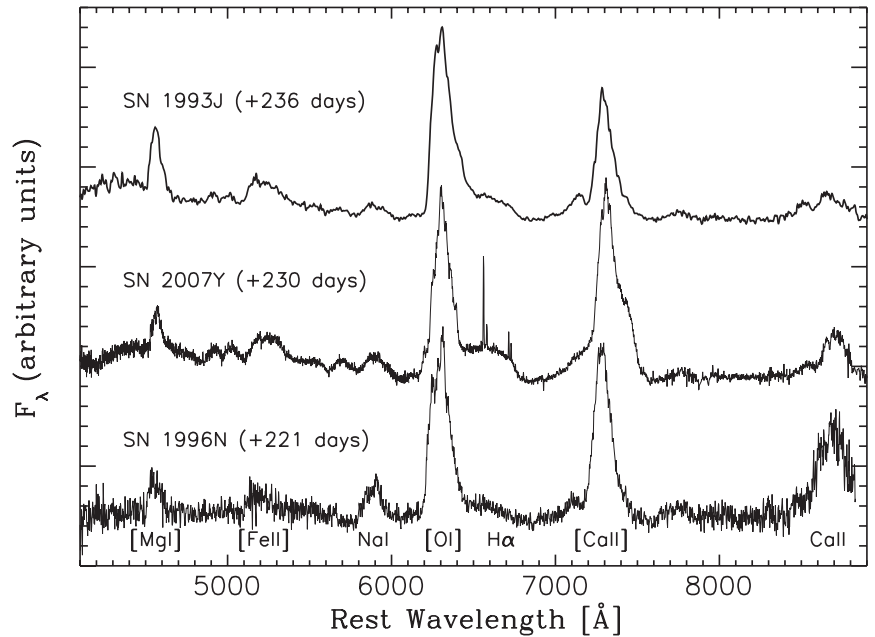

Figure 12. Comparison of similar phase nebular spectra of SN 1993J (Barbon et al. 1994), SN 1996N (Sollerman et al. 1998), and SN 2007Y. The wavelength scale has been corrected for the redshift to the host galaxy of each SN. Prominent emission features are identified.

spectra in Figure 12, is 0.6, 0.9, and 1.0 for SN 1993J, SN 1996N and SN 2007Y, respectively. For perspective, this ratio, was $\approx 0.5$ in SN 1998bw. Fransson \& Chevalier $(1987,1989)$ have shown that the $[\mathrm{Ca} \mathrm{II}] /[\mathrm{O} \mathrm{I}]$ ratio stays relatively constant during the nebular phase, and is a good diagnostic of the core mass, where a higher ratio indicates a less massive core. From stellar evolution it is well known that the core mass is highly dependent on the zero-age-main-sequence mass (ZAMS) of the progenitor. That the $[\mathrm{Ca} \mathrm{II}] /[\mathrm{O} \mathrm{I}]$ ratio in $\mathrm{SN} 2007 \mathrm{Y}$ is $>0.5$ suggests that its progenitor star had a main sequence mass comparable to SNe $1993 \mathrm{~J}$ and $1996 \mathrm{~N}$, i.e. $M_{\mathrm{ZAMS}} \lesssim 20 M_{\odot}$ (Nomoto et al. 1993; Podsiadlowski et al. 1993; Shigeyama et al. 1994). This is much lower than the progenitor of SN 1998bw, which had a main sequence mass $M_{\text {ZAMS }} \approx 40 M_{\odot}$ (Iwamoto et al. 1998; Nakamura et al. 2001). To obtain a more quantitative estimate of the ejecta mass and hence the ZAMS mass of the progenitor we turn to spectral synthesis.

\subsection{Nebular Spectral Synthesis}

To model the nebular spectrum we used a one-zone non-local thermodynamic equilibrium (NLTE) code (Mazzali et al. 2001, 2007a). Heating within the ejecta comes from the deposition of $\gamma$ rays and positrons produced from the decay of ${ }^{56} \mathrm{Co}$ to ${ }^{56} \mathrm{Fe}$. This is balanced by cooling via nebular line emission. The emission rate of each line is determined by solving a NLTE matrix of level populations (Axelrod 1980). The emitting volume is assumed to be of finite extent, homologously expanding, and has a uniform density and composition. The emission spectrum is computed from assigning a parabolic profile to each line which is bounded by the velocity of the outer edge of the ejecta.

The main parameters determining the synthetic spectrum are the width of the emission lines, the mass of radioactive ${ }^{56} \mathrm{Ni}$, and the mass of the other elements. As iron is a product of the decay of ${ }^{56} \mathrm{Ni}$, the width of the [Fe II] emission at 5200 $\AA$ gives an indication of the distribution of ${ }^{56} \mathrm{Ni}$. Abundances of all elements except sulfur and silicon were determined by fitting the emission line profiles. Unfortunately neither of these ions have a strong or isolated emission lines in the optical. Therefore the abundances of these two elements are not strongly constrained.

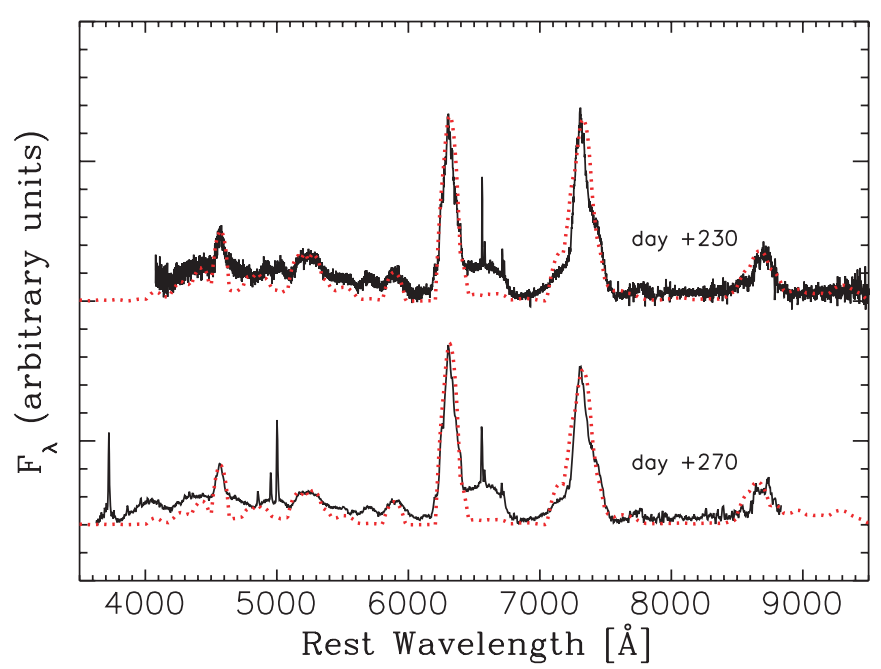

Figure 13. Nebular spectra obtained on day +230 and +270 compared to synthetic spectra (dotted line).

(A color version of this figure is available in the online journal.)

Table 6

Radio Observations of SN 2007Y

\begin{tabular}{lccc}
\hline \hline $\begin{array}{l}\text { Date Obs } \\
(\mathrm{UT})\end{array}$ & $\begin{array}{c}\Delta t^{\mathrm{a}} \\
(\text { days })\end{array}$ & $\begin{array}{c}F_{v, 8.46 \mathrm{GHz}} \\
(\mu \mathrm{Jy})\end{array}$ & $\begin{array}{c}\text { Array } \\
\text { Config. }\end{array}$ \\
\hline 2007 Feb 24.01 & 10 & \pm 34 & $\mathrm{D}$ \\
2007 Mar 10.89 & 25 & \pm 18 & $\mathrm{D}$ \\
2007 May 26.79 & 102 & \pm 37 & $\mathrm{~A}$ \\
2007 Dec 21.21 & 310 & \pm 33 & $\mathrm{~B}$ \\
2008 Jun 13.66 & 486 & \pm 38 & $\mathrm{D}$ \\
2008 Dec 16.22 & 671 & \pm 27 & $\mathrm{~A}$
\end{tabular}

Notes.

a Assuming an explosion date of 2007 February 14 UT or JD-2454145.5.

${ }^{\mathrm{b}}$ All flux densities are given as $1 \sigma$ (rms).

Given the short baseline in time covered by the nebular spectra we elected to model the two best spectra. These observations and the corresponding synthetic spectra are shown in Figure 13. In both cases the $5300 \AA[\mathrm{Fe}$ II] blend is best fitted with a model having an outer velocity of $4500 \mathrm{~km} \mathrm{~s}^{-1}$. This is also true for the $\left[\mathrm{O}_{\mathrm{I}}\right] \lambda 6300$ doublet. Having an identical velocity for these two lines is often the case in low energy SNe Ib/c (e.g., Sauer et al. 2006), and is another indication that the explosion was not strongly aspherical. Both synthetic spectra shown in Figure 13 have a total ejected mass of $0.44 M_{\odot}$ below $4500 \mathrm{~km} \mathrm{~s}^{-1}$. Of this mass $0.06 M_{\odot}$ is ${ }^{56} \mathrm{Ni}$ and $\approx 0.20 M_{\odot}$ is oxygen. The value for the ${ }^{56} \mathrm{Ni}$ abundance confirms the results obtained from both the early and late phase UVOIR light curve.

\subsection{Radio Observations and Circumstellar Interaction}

In Table 6 we summarize our radio observations of SN 2007Y. All observations were conducted with the VLA in the standard continuum mode with a bandwidth of $2 \times 50 \mathrm{MHz}$ centered at $8.46 \mathrm{GHz}$. We used $3 \mathrm{C} 48$ and $3 \mathrm{C} 286(\mathrm{~J} 0137+331$ and J1331+305) for flux calibration, while J0240-231 was used to monitor the phase. Data were reduced using standard packages within the Astronomical Image Processing System (AIPS).

No radio emission was detected at the optical SN position in any of our VLA observations. The initial radio upper limits imply that SN $2007 \mathrm{Y}$ was a factor of $\sim 10^{4}$ less luminous than 


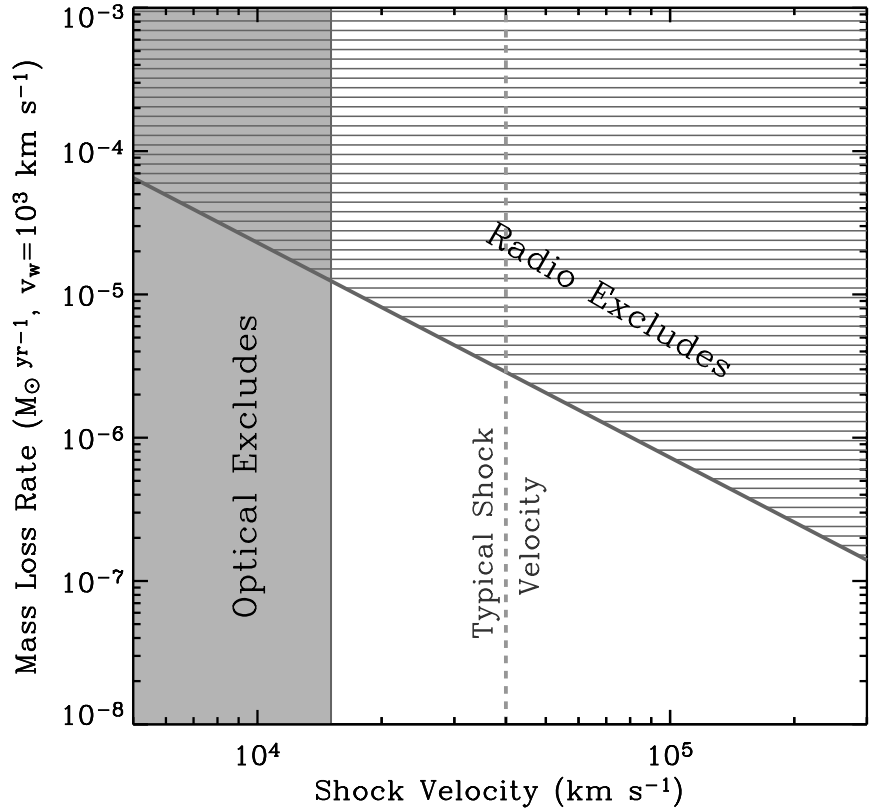

Figure 14. Two-dimensional $v_{s}-\dot{M}$ parameter space. Combining constraints on the photospheric velocity derived from the early phase spectra and the upper limits from radio observations we find a mass-loss rate of the progenitor star to be $\dot{M} \lesssim 10^{-6} M_{\odot} \mathrm{yr}^{-1}$

SN 1998bw on a comparable timescale (Kulkarni et al. 1998). We conclude that SN 2007Y, like the majority of SNe Ib/c, did not produce relativistic ejecta along our line-of-sight (Soderberg et al. 2006). Our later observations at $t \sim 2$ yrs further constrain the presence of a relativistic jet initially directed away from our line of sight. The upper limit on the late-time flux density is $F_{v}<54 \mu \mathrm{Jy}(2 \sigma)$, a factor of $10^{4}$ less luminous than well studied radio afterglows on the same timescale, and following the transition to non-relativistic and spherical Sedov-Taylor expansion (e.g., GRB 030329; Frail et al. 2005).

While optical data probe the thermal emission from the slow moving bulk material, nonthermal synchrotron emission is produced as the forward shock races ahead and dynamically interacts with the CSM. Making the standard assumption that the post-shock energy density is in equipartition between relativistic electrons $\left(\epsilon_{e}\right)$ and amplified magnetic fields $\left(\epsilon_{B}\right)$, the velocity of the radio emitting material is robustly determined (Chevalier 1998). Adopting typical values, $\epsilon_{e}=\epsilon_{B}=0.1$, provides an estimate for the density of the CSM and, in turn, the mass-loss rate of the progenitor star. Together, the velocity of the shock $\left(v_{s}\right)$ and the mass-loss rate of the progenitor $(M)$ determine the luminosity and peak time of the bell-shaped radio light curve.

Since any radio emission from SN 2007Y fell below the VLA detection threshold, we use $(2 \sigma)$ upper limits from Table 6 to constrain the characteristics (luminosity, shape) of the light curve. Adopting the formalism of Chevalier \& Fransson (2006) for the parameterization of the radio light curves of $\mathrm{SNe}$ dominated by synchrotron self-absorption, we use our light curve constraints for SN 2007 Y to place robust constraints on $v_{s}$ and $\dot{M}$. In Figure 14 we show the two-dimensional $v_{s}-\dot{M}$ parameter space that is ruled out by our radio upper limits. With the necessity that the forward shock velocity is faster than the observed photospheric velocity in the homologous outflow, a second region is excluded. Combining these constraints we find that the mass-loss rate of the SN $2007 \mathrm{Y}$ progenitor star is less than $\dot{M} \approx 10^{-5} M_{\odot} \mathrm{yr}^{-1}$ where we have assumed a progenitor wind velocity of $v_{w}=1000 \mathrm{~km} \mathrm{~s}^{-1}$, appropriate for Wolf-Rayet stars. Adopting a typical shock velocity of $v_{s} \approx 40,000 \mathrm{~km} \mathrm{~s}^{-1}$ consistent with well studied radio $\mathrm{SNe}$ $\mathrm{Ib} / \mathrm{c}$, implies that $\dot{M} \lesssim 10^{-6} M_{\odot} \mathrm{yr}^{-1}$ which is on the low end of the observed values for radio $\mathrm{SNe} \mathrm{Ib} / \mathrm{c}$ (Chevalier \& Fransson 2006). Finally we estimate the mass of CSM swept up by the forward shock, $M_{\mathrm{CSM}}$, over the course of our radio observations to be less than $M_{\mathrm{CSM}} \approx 2 \times 10^{-5} M_{\odot}$ assuming a standard stellar wind density profile.

\section{DISCUSSION}

The observational properties of SN 2007Y have a number of qualities in common with other Type Ib and Type IIb SNe. Spectra obtained prior to maximum light closely resemble early spectra of SNe 1999ex and 2005bf, while at late times SN 2007Y has commonalities with SNe 1993J and 1996N. Nevertheless, subtle differences between these events and SN 2007Y do exist and reflects, among other things, variations in the composition and mass of the progenitor, the kinetic energy of the explosion, and the degree of asphericity of the ejecta.

The early spectra exhibit the infamous $6200 \AA$ absorption line that is often, but not always, observed in $\mathrm{SNe} \mathrm{Ib/c} \mathrm{(Matheson}$ et al. 2001). Currently, it is a matter of open debate which ion(s) form this feature. Previous authors have proposed a number of sources that range from $\mathrm{H} \alpha$ (Branch et al. 2002; Anupama et al. 2005; Folatelli et al. 2006; Parrent et al. 2007; Pastorello et al. 2008) or Si II $\lambda 6355$ (e.g., Hamuy et al. 2002), to Si II blended with $\mathrm{H} \alpha$ (Tanaka et al. 2009). Others have invoked C II $\lambda 6580$ blended with $\mathrm{H} \alpha$ (Deng et al. 2000) or only Ne I $\lambda 6402$ (Benetti et al. 2002). Recently, more detailed calculations reported by Ketchum et al. (2008) suggest Si II blended with Fe II as a viable source. In the case of SN 2007 Y we lean toward an identification of $\mathrm{H} \alpha$ possibly blended with $\mathrm{Si}$ II. The hydrogen hypothesis is supported in part by the $\mathrm{H} \alpha$ emission in the nebular spectrum that, similar to early epochs, is at a higher velocity than the other material. Additional evidence for hydrogen comes from a notch in the earliest spectra (see Figure 10) which may be due to $\mathrm{H} \beta$ blueshifted by $\sim 14,000 \mathrm{~km} \mathrm{~s}^{-1}$. With these pieces of evidence, and the arguments laid out by Anupama et al. (2005), Folatelli et al. (2006) and Parrent et al. (2007) for the case of SN 2005bf, we conclude that $\mathrm{H} \alpha$ is likely the dominant ion responsible for the absorption at $6200 \AA$. The gross inconsistency between the blueshift derived from Si II, compared to the other ions (see Figure 9), allows us to reject Si II as the only ion producing this feature. To decisively ascertain to what extent these or other ions attribute to the $6200 \AA$ feature remains to be determined through sophisticated radiative transfer calculations.

The identification of high-velocity calcium gas has also been reported in the early phase spectra of SN 1999ex and SN 2005bf. The strong spectroscopic similarity of these events to SN 2007Y, and the fact that by maximum light both SN 1999ex and SN 2007Y are representative of a normal Type Ib event are both suggestive that this may be a generic feature of many SNe Ib. The fast evolution of the Ca II triplet highlights the importance to obtain premaximum spectra of these events in order to confirm if high-velocity calcium gas is in fact a commonality among other $\mathrm{SNe} \mathrm{Ib} / \mathrm{c}$. Note, however, there appears to be no signature of this feature in the early epoch spectra of SN 2008D.

We estimate $0.06 M_{\odot}$ of ${ }^{56} \mathrm{Ni}$ was synthesized in the explosion. We also find from modeling the nebular spectra a lower 

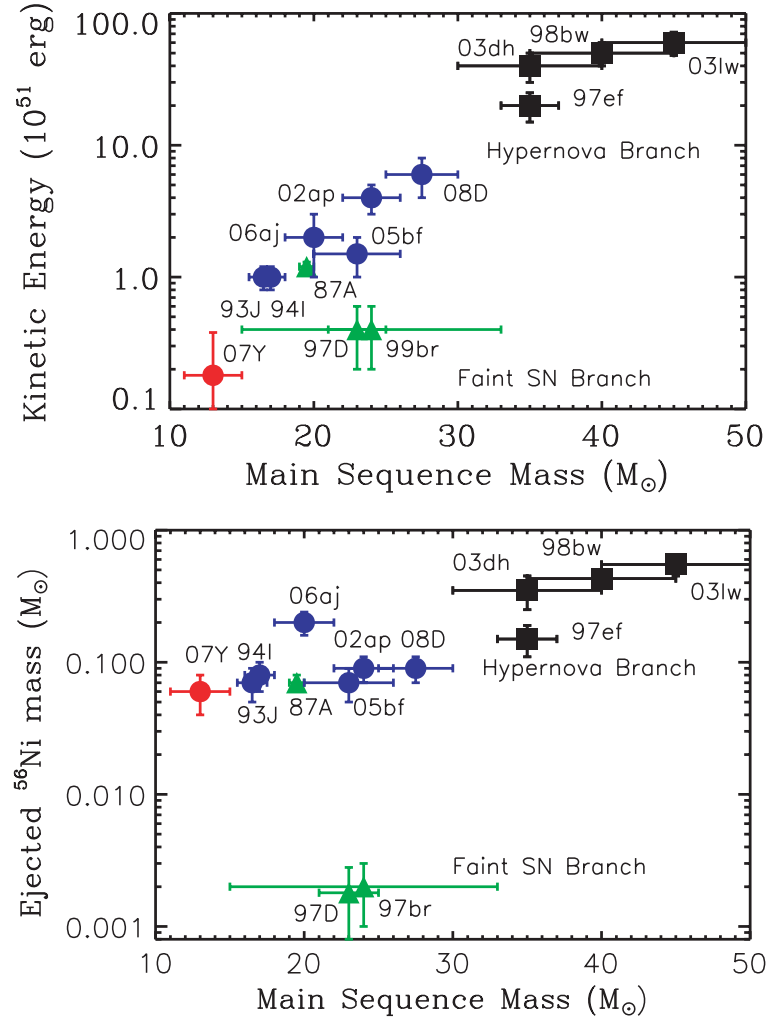

Figure 15. Comparison of physical parameters for a set of core collapse SNe. The upper panel is the kinetic energy of the explosion and the lower panel is the ejected ${ }^{56} \mathrm{Ni}$-mass vs. the estimated ZAMS mass of the progenitor stars. Blue filled circles are normal Type Ib/c SNe, black filled squares are Type Ic hypernovae, and green filled triangles are Type II SNe. Parameters of the comparison SNe are taken from the literature; SN 1987A: standard values; SN 1993J: standard values; SN 1994I: Sauer et al. (2006); SN 1997D: Turatto et al. (1998); SN 1997ef: Mazzali et al. (2004); SN 1998bw: Mazzali et al. (2001); SN 1999br: Zampieri et al. (2003); SN 2002ap Mazzali et al. (2007b); SN 2003dh: Mazzali et al. (2001); SN 20031w: Mazzali et al. (2006a); SN 2005bf: Tominaga et al. (2005); SN 2006aj: Mazzali et al. (2006b); SN 2008D: Mazzali et al. (2008).

(A color version of this figure is available in the online journal.)

limit on the ejected oxygen mass below $4500 \mathrm{~km} \mathrm{~s}^{-1}$ of $0.20 M_{\odot}$, and a total ejecta mass of $0.44 M_{\odot}$. Stellar evolutionary models predict this amount of oxygen is produced from He stars with a mass of $\sim 3.3 M_{\odot}$ (Thielemann et al. 1996; Woosley et al. 1995; Shigeyama et al. 1990). The He star most likely evolved from a ZAMS star of $\approx 10-13 M_{\odot}$ that formed in a binary system, and during its evolution, was able to shed its envelope through stable case C mass transfer (for an example see Podsiadlowski et al. 1993). In this scenario, prior to explosion, the He star retains a residual hydrogen shell with a mass of a few tenths of a solar mass or less. Alternatively, but more unlikely given the abundance pattern, the progenitor star could have been a massive star $\left(M_{\text {ZAMS }}>35 M_{\odot}\right)$ that underwent strong stellar winds prior to explosion (Woosley et al. 1993). A scenario of a more massive star is also disfavored given the lack of evidence for asymmetry in the ejecta.

Now we attempt to estimate the kinetic energy of the explosion. As previously discussed in Section 3.3, the similarity between the light curve shape of SNe 2006aj and 2007Y implies $M_{\mathrm{ej}}(07 \mathrm{Y})^{3} / E_{\mathrm{KE}}(07 \mathrm{Y}) \propto M_{\mathrm{ej}}(06 \mathrm{aj})^{3} / E_{\mathrm{KE}}(06 \mathrm{aj})$. Plugging in $M_{\mathrm{ej}}(06 \mathrm{aj})=1.8 M_{\odot}, E_{\mathrm{KE}}(06 \mathrm{aj})=2.0 \times 10^{51} \mathrm{erg}$ (Maeda et al. 2007a; Mazzali et al. 2006b) and $M_{\mathrm{ej}}(07 \mathrm{Y})=0.50 M_{\odot}$, and then solving for $E_{\mathrm{KE}}(07 \mathrm{Y})$, gives $\approx 4.3 \times 10^{49} \mathrm{erg}$. Alternatively, simply looking at the ejecta mass and the velocity of the nebula $E_{\mathrm{KE}}(07 \mathrm{Y}) \approx(1 / 2) \cdot M_{\mathrm{ej}}(07 \mathrm{Y}) \cdot v_{\mathrm{neb}}^{2} \approx 1.0 \times 10^{50} \mathrm{erg}$.

A more accurate approach is to combine the fact that the light curve shapes of SN 2007Y and SN 2006aj are similar to the ratio of their photospheric expansion velocity via $v_{\mathrm{ph}} \propto$ $\left(E_{\mathrm{KE}} / M_{\mathrm{ej}}\right)^{1 / 2}$. At maximum light the ratio of the photospheric velocity of SN 2007Y $\left(v_{\mathrm{ph}}=9000 \mathrm{~km} \mathrm{~s}^{-1}\right)$ to $\mathrm{SN} 2006 \mathrm{j}$ $\left(v_{\mathrm{ph}}=15,000 \mathrm{~km} \mathrm{~s}^{-1}\right.$; Pian et al. 2006) is 0.60 . This implies a $E_{\mathrm{KE}}(07 \mathrm{Y}) \sim 1.8 \times 10^{50} \mathrm{erg}$. If we double $M_{\mathrm{ej}}(07 \mathrm{Y})$ then $E_{\mathrm{KE}}(07 \mathrm{Y})$ increases by a factor of 2 . A $E_{\mathrm{KE}}(07 \mathrm{Y})$ of $\approx$ few $\times$ $10^{50} \mathrm{erg}$ is nearly an order of magnitude less than the $\geqslant 10^{51} \mathrm{erg}$ that is found in other $\mathrm{SNe} \mathrm{Ib/c.}$

In Figure 15 the physical parameters of SN 2007Y are compared to those of 13 other well studied core-collapse SNe. Here the estimated values of $M_{\text {ZAMS }}$ are compared to (top panel) $E_{\mathrm{KE}}$ and (bottom panel) the ejected ${ }^{56} \mathrm{Ni}$ mass. From its position in the top panel we find SN 2007Y to be one of the least energetic $\mathrm{SNe} \mathrm{Ib} / \mathrm{c}$ yet studied. Correspondingly, the ${ }^{56} \mathrm{Ni}$ mass is comparable to the least luminous $\mathrm{SNe} \mathrm{Ib} / \mathrm{c}$, while the $M_{\mathrm{ZAMS}}$ lies at the low end of the $M_{\mathrm{ZAMS}}$ distribution of the comparison sample, most similar to SN 1993J.

\section{CONCLUSION}

We present detailed photometric and spectroscopic observations of SN 2007Y. SN 2007Y appears to be one of the least energetic normal $\mathrm{SN}$ Ib yet studied, with $E_{\mathrm{KE}} \sim 10^{50} \mathrm{erg}$. We estimate a total ejected mass of $0.45 M_{\odot}$, of which $0.06 M_{\odot}$ is ${ }^{56} \mathrm{Ni}$ and $0.2 M_{\odot}$ is $\mathrm{O}$. The oxygen abundance suggests the progenitor was most likely a $3.3 M_{\odot}$ He-core star that evolved from a 10-13 $M_{\odot}$ main-sequence mass star in a binary system. These physical parameters all point toward a progenitor similar to that of SN 1993J.

The remarkable similarity of the early phase spectra of SN 2007Y compared to SN 1999ex and SN 2005bf, suggests these events had very similar photospheric conditions. However, subtle difference do exist. These are most likely related to differences in the total ejected mass and the configuration of the ejecta. In the earliest phases we identify high-velocity features of $\mathrm{H} \alpha$ and $\mathrm{Ca}$ II. These features may be a commonality in many other $\mathrm{SNe} \mathrm{Ib} / \mathrm{c}$. This remains to be confirmed with early phase observations that are expected to be performed in future all-sky surveys.

This material is based upon work supported by the National Science Foundation (NSF) under grant AST-0306969. The Dark Cosmology Centre is funded by the Danish NSF. M.S. acknowledges support from the MPA's visitor programme. J.S. is a Royal Swedish Academy of Sciences Research Fellow supported by a grant from the Knut and Alice Wallenberg Foundation. M.H. ackowledges support from Iniciativa Cientifica Milenio through grant P06-045-F and CONICYT through Centro de Astrofisica FONDAP (grant 15010003), Programa Financiamiento Basal (grant PFB 06), and Fondecyt (grant 1060808). We have made use of the NASA/IPAC Extragalactic Database (NED) which is operated by the Jet Propulsion Laboratory, California Institute of Technology, under contract with the National Aeronautics and Space Administration. This publication makes use of data products from the Two Micron All Sky Survey, which is a joint project of the University of Massachusetts and the Infrared Processing and Analysis Center/California Institute of Technology, funded by the National Aeronautics and Space Administration and the National Science Foundation. 


\section{APPENDIX}

\section{OBSERVATIONS AND DATA REDUCTION}

\section{A.1. Photometry}

The UVOT light curves were originally presented in Brown et al. (2008), and the reader is referred to that paper and references therein for details of the data acquisition and reduction process. The light curves have superb sampling that cover the evolution of SN 2007Y from - 11.6 to 21.6 days past maximum light.

Twenty-one epochs of early-time optical $\left(u^{\prime} g^{\prime} r^{\prime} i^{\prime} B V\right)$ imaging were acquired with the LCO's Henrietta Swope $1 \mathrm{~m}$ telescope equipped with a direct imaging CCD camera, and two epochs of spectroscopic acquisition imaging with the du Pont (+WFCCD: Wide Field Re-imaging CCD Camera) $2.5 \mathrm{~m}$ telescope. We direct the reader to Hamuy et al. (2006, Paper I) and C. Contreras et al. (2009, in preparation) for a detailed technical description of the telescopes, instruments, and filter transmission functions used in the low- $z$ portion of the CSP. Paper I also contains a step by step account of the data reduction process for each instrument used by the CSP.

The brightness of SN 2007Y was determined differentially with respect to a sequence of local field stars. Absolute photometry of the local sequence in the optical (Table 1) was obtained using standard fields (Landolt 1992; Smith et al. 2002) observed on four photometric nights.

Instrumental magnitudes of the standard fields were computed using the IRAF ${ }^{23}$ DAOPHOT package PHOT with an aperture radius of $7^{\prime \prime}$. Adopting extinction coefficients and color terms derived through the course of the CSP (see Figure 3 and Figure 4 in Paper I) the instrumental magnitudes of the standard stars were used to derive zero points to calibrate the local sequence.

The magnitude of SN 2007Y and the local sequence were measured by a point-spread function (PSF) technique. A PSF within a radius of $3^{\prime \prime}$ was fitted to each sequence star and the SN. A night-to-night zero point was computed from the local sequence and then used to obtain the apparent magnitude of SN 2007Y.

Although the SN was located well outside the spiral arms, close inspection of optical VLT images obtained under excellent seeing conditions revealed the presence of an $\mathrm{H}$ II region adjacent to the location of SN 2007Y. To assess if our photometry was contaminated by either this $\mathrm{H}$ II region or any spatial/time varying background, optical galaxy templates of NGC 1187 were obtained on March 5.2, 2008 with a direct imaging camera (Tek 5) attached to the du Pont. These templates were used to subtract any background on all early-time CSP $u^{\prime} g^{\prime} r^{\prime} i^{\prime} B V$ images. We found mean magnitude differences for each light curve (in the sense of photometry computed with versus without template subtraction) of $\Delta\left(u^{\prime}, g^{\prime}, r^{\prime}, i, B, V\right)=$ $(0.009,0.003,0.004,0.001,0.003,0.003)$. These small differences indicate that no systematic offset was introduced to the images from template subtraction and that any background in the optical is negligible.

Two epochs of late-time $B V R I$ imaging at 270 and 343 days past $L_{\max }$ were obtained with the VLT (+FORS1) in service mode. Science product images reduced by the ESO pipeline were used for the aperture photometry. As all of the local sequence stars used in the early phase imaging were saturated in the VLT images it proved necessary to define another local sequence. Absolute photometry of this local sequence was obtained using the standard star field PG2331+055 (Landolt 1992) observed during the first photometric epoch of VLT imaging. Instrumental magnitudes of the 3 stars in PG2331+055 were computed using PHOT with an aperture radius of $7^{\prime \prime}$. These instrument magnitudes were then corrected for atmospheric extinction and color terms using standard values derived for Chip 1 of FORS1. ${ }^{24}$ Zero points were then computed by comparing the instrumental magnitudes to the Landolt standard magnitudes. Instrumental magnitudes of seven local sequence stars (and SN 2007Y) were then computed with a 0.'5 aperture radii plus an aperture correction. After color and atmospheric extinction corrections the zero points derived from PG2331+055 were applied to these magnitudes to calibrate the VLT local sequence. This local sequence was then used to determine nightly zero points in order to obtain apparent magnitudes of SN 2007Y.

Fifteen epochs of early phase near-infrared photometry were acquired at LCO with the Swope (+RetroCam) and du Pont (+WIRC: Wide field InfraRed Camera) telescopes. The majority of $Y J H$-band imaging was taken with RetroCam, while several epochs including the $K_{s}$ band were obtained with the WIRC.

Imaging was generally performed with a jitter technique that consists of a two loops of nine exposures. Each of the individual images were dark-subtracted, flat-fielded and then stacked to produce a master image used to calculate photometry.

Instrumental magnitudes of standard star fields (Persson et al. 1998) observed over 5 photometric nights were measured with an aperture radius of $5^{\prime \prime}$. Zero points were then derived from these observations in order to calibrate a local sequence whose instrumental magnitudes were computed via a small aperture and aperture correction. Standard extinction coefficients (Persson et al. 1998) were used and no color term corrections were applied. In the $K_{s}$-band photometry we were unable to calibrate a local sequence due to a lack of standard field observations. Fortunately four of the stars that make up our local sequence were also observed by the Two Micron All Sky Survey (2MASS) survey. We therefore adopted 2MASS magnitudes for these stars and the corresponding values are reported in Table 1. Finally, differential photometry of SN 2007Y (Table 3) was obtained relative to the local sequence.

Late epoch imaging was performed with the Infrared Spectrometer And Array Camera (ISAAC) attached to the VLT. Imaging was performed in the short wavelength mode with multiple loops of the jitter-offset mode. Reduction of this data was performed with the Eclipse software package (Devillard 1997). The jitter program was used to estimate and remove the sky background from each on source image. These were then combined to produce final stacked images that were used to derive instrumental magnitudes. Absolute photometry was determined using several Persson standard star fields observed over the course of the night the SN was monitored. We were only able to measure the brightness of SN 2007Y in the $J_{\mathrm{S}}$ and $H$ band in the first epoch of near-infrared imaging, i.e., day +268 . In the cases when the $\mathrm{SN}$ was not detected a $3 \sigma$ upper limit of its brightness was derived.

\footnotetext{
23 The image Reduction and Analysis Facility (IRAF) is maintained and distributed by the Association of Universities for Research in Astronomy, under the cooperative agreement with the National Science Foundation.
}

\footnotetext{
${ }^{24}$ http://www.eso.org/observing/dfo/quality/FORS1/qc/photcoeff/ photcoeffs_fors $1 . h t m l$
} 


\section{A.2. Spectroscopy}

Twelve epochs of long slit spectroscopy was obtained from -14 to +270 days past $L_{\max }$. All spectra were reduced in the same manner using standard techniques as described in Paper I. In summary, each spectrum was overscan subtracted, bias corrected, trimmed, flat-fielded, and then extracted. An accurate wavelength calibration solution was derived from arc lamp spectra and applied to the extracted one-dimensional spectrum. Finally, a nightly response function derived from standard star observations was used to flux-calibrate each spectrum. When multiple spectra were obtained for a given epoch they were combined to produce a master spectrum.

\section{REFERENCES}

Anupama, G. C., et al. 2005, ApJ, 631, L125

Arnett, W. D. 1982, ApJ, 253, 785

Axelrod, T. S. 1980, PhD thesis, Univ. California, Santa Cruz

Barbon, R., et al. 1994, A\&AS, 110, 513

Benetti, S., et al. 2002, MNRAS, 336, 91

Branch, D., et al. 2002, ApJ, 566, 1005

Brown, P., et al. 2008, AJ, submitted (arXiv:0803.1265)

Burrows, D. N., et al. 2005, Space Sci. Rev., 120, 165

Chevalier, R. A. 1998, ApJ, 499, 810

Chevalier, R. A., \& Fransson, C. 1994, ApJ, 420, 268

Chevalier, R. A., \& Fransson, C. 2006, ApJ, 651, 381

Clocchiatti, A., \& Wheeler, C. 1994, IAU Circ. 6005

Clocchiatti, A., et al. 2008, PASP, 120, 290

Deng, J. S., et al. 2000, ApJ, 540, 452

Devillard, N. 1997, ESO Messenger, 87, 19

Dickey, J. M., \& Lockman, F. J. 1990, ARA\&A, 28, 215

Elmhamdi, A., et al. 2004, A\&A, 426, 963

Filippenko, A. V., Matheson, T., \& Barth, A. J. 1994, AJ, 108, 2220

Folatelli, G., Morrell, N., Phillips, M., \& Hamuy, M. 2007, CBET, 862

Folatelli, G., et al. 2006, ApJ, 641, 1039

Frail, D. A., et al. 2005, ApJ, 619, 994

Fransson, C., \& Chevalier, R. A. 1987, ApJ, 322, 15

Fransson, C., \& Chevalier, R. A. 1989, ApJ, 343, 323

Fransson, C., Lundqvist, P., \& Chevalier, R. A. 1996, ApJ, 461, 993

Freedman, W. L., et al. 2001, ApJ, 553, 47

Fynbo, J. P. U., et al. 2004, ApJ, 609, 962

Galama, T. J., et al. 1998, Nature, 395, 670

Hamuy, M., et al. 2002, AJ, 124, 417

Hamuy, M., et al. 2006, PASP, 118, 2

Hillenbrand, L. A., Foster, J. B., Persson, S. E., \& Matthews, K. 2002, PASP, 114,708

Houch, J. C., \& Fransson, C. 1996, ApJ, 456, 811

Hjorth, J., et al. 2003, Nature, 423, 847

Immler, S., et al. 2006, ApJ, 648, L119

Immler, S., et al. 2007, ApJ, 664, 435

Iwamoto, K., et al. 1998, Nature, 395, 672

Ketchum, W., Baron, E., \& Branch, D. 2008, ApJ, 674, 371

Kulkarni, S. R., et al. 1998, Nature, 395, 663

Landolt, A. U. 1992, AJ, 104, 340

Maeda, K., et al. 2007a, ApJ, 658, L5

Maeda, K., et al. 2007b, ApJ, 666, 1069

Maeda, K., et al. 2008, Science, 319, 1220
Malesani, D., et al. 2004, ApJ, 609, L5

Malesani, D., et al. 2009, ApJ, 692, L84

Matheson, T., et al. 2000, AJ, 120, 1487

Matheson, T., et al. 2001, AJ, 121, 1648

Matheson, T., et al. 2003, ApJ, 599, 394

Maund, J., et al. 2007, MNRAS, 381, 201

Mazzali, P. A., et al. 2001, ApJ, 559, 1047

Mazzali, P. A., et al. 2003, ApJ, 599, 95

Mazzali, P. A., et al. 2004, ApJ, 614, 858

Mazzali, P. A., et al. 2006, ApJ, 645, 1323

Mazzali, P. A., et al. 2006, Nature, 442, 1018

Mazzali, P. A., et al. 2007, ApJ, 661, 892

Mazzali, P. A., et al. 2007, ApJ, 670, 592

Mazzali, P. A., et al. 2008, Science, 321, 1185

Mirabal, N., et al. 2006, ApJ, 643, L99

Modjaz, M., et al. 2006, ApJ, 645, L21

Modjaz, M., et al. 2009, ApJ, submitted

Monard, L. A. G. 2007, IAU Circ. 8813

Nakamura, T., et al. 2001, ApJ, 550, 991

Nomoto, K., Suzuki, T., Shigeyama, T., Yamaoka, H., \& Saio, H. 1993, Nature, 364,507

Parrent, J., et al. 2007, PASP, 119, 135

Patat, F., Chugai, N., \& Mazzali, P. A. 1995, A\&A, 299, 715

Patat, F., et al. 2001, ApJ, 555, 900

Pastorello, A., et al. 2008, MNRAS, 389, 955

Pei, Y. C. 1992, ApJ, 395, 130

Persson, S. E., et al. 1998, AJ, 116, 2475

Podsiadlowski, P., Hsu, J. J. L., Joss, P. C., \& Ross, R. R. 1993, Nature, 364 509

Pian, E., et al. 2006, Nature, 442, 1011

Poole, T. S., et al. 2008, MNRAS, 383, 627

Richardson, D., Branch, D., \& Baron, E. 2006, AJ, 131, 2233

Richmond, M. W., et al. 1994, AJ, 107, 1022

Richmond, M. W., et al. 1996, AJ, 111, 327

Roming, P. W. A., et al. 2005, Space Sci. Rev., 120, 95

Sauer, D., et al. 2006, MNRAS, 369, 1939

Schlegel, D. J., Finkbeiner, D. P., \& Davis, M. 1998, ApJ, 500, 525

Shigeyama, T., Nomoto, K., Tsujimoto, T., \& Hashimoto, M. 1990, ApJ, 361, L23

Shigeyama, T., et al. 1994, ApJ, 420, 341

Smith, J. A., et al. 2002, AJ, 123, 2121

Soderberg, A. M, Berger, E., Ofek, E., \& Leonard, D. C. 2005, ATEL, 646, 1

Soderberg, A. M., et al. 2006, ApJ, 638, 930

Soderberg, A. M., et al. 2008, Nature, 453, 469

Sollerman, J., Leibundgut, B., \& Spyromilio, J. 1998, A\&A, 337, 207

Sollerman, J., et al. 2006, A\&A, 454, 503

Stanek, K. Z., et al. 2003, ApJ, 591, L17

Stritzinger, M., et al. 2002, AJ, 124, 2100

Stritzinger, M., et al. 2005, PASP, 117, 810

Tanaka, M., et al. 2009, ApJ, 692, 1131

Taubenberger, S., et al. 2006, MNRAS, 371, 1459

Thielemann, F. K., Nomoto, K., \& Hashimoto, M. 1996, ApJ, 460, 408

Tominaga, N., et al. 2005, ApJ, 633, L97

Tomita, H., et al. 2006, ApJ, 644, 400

Turatto, M., et al. 1998, ApJ, 498, 129

Turatto, M., Benetti, S., \& Cappellaro, E. 2003, in From Twilight to Highlight: the Physics of Supernovae, ed. B. Leibundgut, W. Hillebrandt (Berlin: Springer), 200

Woosley, S. E., Langer, M., \& Weaver, T. A. 1993, ApJ, 411, 823

Woosley, S. E., Langer, M., \& Weaver, T. A. 1995, ApJ, 448, 315

Zampieri, L., et al. 2003, MNRAS, 338, 711 